\title{
Capture numbers in the presence of repulsive adsorbate interactions
}

\author{
J. A. Venables ${ }^{1, *}$ and H. Brune ${ }^{2}$ \\ ${ }^{1}$ Department of Physics and Astronomy, Arizona State University, Tempe, Arizona 85287-1504 \\ and CPES, University of Sussex, Brighton, United Kingdom \\ ${ }^{2}$ Institut de Physique des Nanostructures, Ecole Polytechnique Fédérale de Lausanne, CH-1015 Lausanne, Switzerland
}

(Received 28 March 2002; published 8 November 2002)

\begin{abstract}
Capture numbers are used in models of nucleation and growth on surfaces, and have been widely applied to predict nucleation densities and other quantities via rate equations. In conventional nucleation theory, much effort has historically been expended on obtaining good expressions for capture numbers in the diffusionlimited case. However, recent experiments and calculations have shown that weak repulsive interactions between adsorbate atoms on relatively smooth (e.g., close-packed metal) surfaces may shift nucleation kinetics towards the attachment-limited case. This paper clarifies the distinctions between diffusion- and attachmentlimited kinetics, and emphasizes the increased importance of the transient nucleation regime in the latter case, which is due to a combination of delayed nucleation and reduced capture. The consequences of long-range repulsive adsorbate interactions for the form and values of the capture numbers are explored, and the effects of attachment-limited kinetics in relation to low-temperature deposition and annealing experiments are demonstrated. An approximate interpolation scheme between attachment- and diffusion-limited kinetics is proposed, and tested against kinetic Monte Carlo simulations. Using this scheme to interpret recent scanning-tunneling microscopy results on $\mathrm{Cu} / \mathrm{Cu}(111)$, lower and upper bounds on the maximum adatom-adatom potential repulsive energy of 10 and $14 \mathrm{meV}$ are deduced.
\end{abstract}

DOI: 10.1103/PhysRevB.66.195404

PACS number(s): 68.55.Ac, 82.20.Kh, 82.20.Pm, 81.15.Kk

\section{INTRODUCTION}

The processes involved in nucleation and growth on surfaces have received widespread attention over the last thirty years. It is now well known that individual atomic events can strongly influence and even dominate the final micro- or nanostructure of epitaxial thin films. ${ }^{1}$ Scanning-tunneling microscopy $^{2}$ (STM) and field-ion microscopy ${ }^{3,4}$ (FIM) experiments are able to follow such individual events. The data obtained on uniform (single-crystal) substrates can be analyzed in detail to obtain diffusion mechanisms, and among other quantities, energies for adsorption $\left(E_{a}\right)$, diffusion $\left(E_{d}\right)$, and binding $\left(E_{b}\right)$ of adatoms.

Rate equations have been used successfully to analyze data, notably of the nucleation density $n_{x}$, as a function of experimental variables, usually the flux $F$ (or equivalently, deposition rate $R$ ) and the substrate temperature $T$. The reaction rates in each equation, e.g., for the single-adatom density $n_{1}$, are of the form $2 \sigma_{1} D_{1} n_{1}^{2}$ (for the rate of adatoms forming pairs) or $\sigma_{x} D_{1} n_{1} n_{x}$ (for the rate of adatoms joining stable clusters). In these terms $\sigma_{1}$ and $\sigma_{x}$ are capture numbers, the subject of this paper, and $D_{1}$ is the single-adatom diffusion coefficient.

Throughout the whole field of materials science, there are typically two extreme types of kinetics: diffusion-limited and attachment-limited. ${ }^{5}$ The purpose of the present paper is to examine capture numbers used for nucleation and growth on surfaces, and to derive quantitative expressions for both limits and interpolation schemes for intermediate cases. In particular, several recent STM experiments at low temperatures on smooth metal surfaces ${ }^{6,7}$ and associated $a b$ initio calculations ${ }^{8,9}$ have highlighted attachment-limited behavior, due to the presence of repulsive barriers between adatoms. Here it shown that these effects modify the results of con- ventional (sometimes called classical) nucleation theory (CNT), by extending the transient nucleation regime to higher dose. New formulas are given for the capture numbers on the assumption of radial symmetry. These expressions are tested against kinetic Monte Carlo (KMC) simulations. As a result, the maximum repulsive interaction energies can be reliably extracted from recent experiments on close-packed metal surfaces; here we concentrate on $\mathrm{Cu} / \mathrm{Cu}(111)$, but the methods developed may also be applied to other systems.

\section{MEAN-FIELD SELF-CONSISTENT CAPTURE NUMBERS}

Capture numbers for particular processes express the probability of the corresponding reaction, and need to be evaluated self-consistently in order to obtain quantitative solutions to models. The need for self-consistency arises because adatom-adatom, adatom-cluster, and cluster-cluster correlations, all forms of self-organization, appear as growth proceeds; this necessity was recognized in early papers ${ }^{10,11}$ and continues to attract much interest.

Two diffusion solutions were found almost thirty years ago, ${ }^{10}$ the first being the uniform depletion approximation; here the other clusters that deplete the adatom concentration around a given cluster are uniformly distributed. The other solution, where the clusters were placed regularly on a grid, leads to the lattice approximation. ${ }^{12}$ In time, it was realized that the uniform depletion solution was in fact a mean-field approximation, analogous to such approximations used in many other fields; this label has often been used since.

The self-consistent label was introduced more recently, in an important paper that compared KMC simulations with rate equations. ${ }^{13}$ In this paper, which discussed the case when adatom pairs are always stable (the critical nucleus size 
$i=1$ ), the comparison was excellent for the monomer and nucleation densities. In particular, as we emphasize below, the mean-field self-consistent capture numbers are the same as those produced by the uniform depletion approximation for this restrictive case, when no complicating factors [adatom evaporation, small cluster instability $(i>1)$, or cluster diffusion] are considered. As shown earlier, ${ }^{10}$ an exact solution for the capture number is available when evaporation is dominant. Moreover, it was also shown that intermediate cases could be treated by defining the adatom lifetime $\tau$, where the various components of $\tau$ add inversely. This idea of competitive capture ${ }^{1,14}$ was then developed quantitatively to allow for individual processes in the equation for the adatom density $n_{1}$, namely,

$$
d n_{1} / d t=F(1-Z)-n_{1} / \tau
$$

with

$$
\tau^{-1}=\tau_{a}^{-1}+\tau_{n}^{-1}+\tau_{c}^{-1}+\ldots .
$$

Here $\tau_{a}$ is the adsorption lifetime, and $\tau_{n}$ the nucleation lifetime, and $\tau_{c}$ the lifetime due to capture of monomers by stable clusters; $Z$ is the coverage of the substrate by (stable and unstable) clusters, not including monomers. The advantage of this formulation is that other processes [ . ., in Eq. (1)] can be added as required in the same fashion.

If evaporation is not important, we may divide through by $F$, so that the independent variable becomes $\theta=F t$, the dose deposited on the substrate. For the case of $i=1$, we have

$$
d n_{1} / d \theta=(1-Z)-\left(2 \sigma_{1} n_{1}^{2}+\sigma_{x} n_{1} n_{x}\right) .\left(D_{1} / F\right) .
$$

Comparing Eq. (2) with Eq. (10) of Ref. 13, we see that they are the same, since the stable cluster density $\sigma_{x} n_{x}$ is the sum of $\sigma_{s} n_{s}$ for all sizes $s \geqslant 2$. We have included in Eq. (2) the main direct impingement (called direct capture in Ref. 13) term, resulting from deposition onto stable clusters, but we have omitted the other one, resulting from deposition onto monomers, for simplicity. Both are generally unimportant at low dose [see, e.g., Fig. 1(b) of Ref. 2). For a discussion of direct impingement see Ref. 10, where the subscript $s$ is $k$, and Ref. 12.

Following Refs. 1, 10, and 14, we may write the nucleation rate of stable clusters in the general case as

$$
d n_{x} / d t=\sigma_{i} D_{1} n_{1} n_{i}-U_{c},
$$

where $n_{i}$ is the density of critical clusters. By transforming to $\theta$ we obtain the nucleation rate for critical size $i$,

$$
d n_{x} / d \theta=\sigma_{i}\left(D_{1} / F\right) C_{i} n_{1}^{(i+1)} \exp \left(\beta E_{i}\right)-U_{c} / F,
$$

where $\beta=(k T)^{-1}$, and the Walton equilibrium relation has been used to express $n_{i}$ in terms of $n_{1}$ and the cluster energy $E_{i} ; C_{i}$ is a statistical weight, and $U_{c}$ is a term due to coalescence of islands. ${ }^{1,10,14}$ When $i>1$, the nucleation term in Eq. (2) needs some modification, as it represents the loss of adatoms in the nucleation event itself; often this term is unimportant numerically, but we need to retain it here. The
CNT adatom and nucleation densities follow from the interplay of Eqs. (2) and (4), or equivalently, the general forms (1) and (3).

The above nucleation equations need to be coupled with some form of cluster growth rate equation [e.g., uniform growth of circular two-dimensional (2D) or 3D islands $s^{1,10,14,15}$ ] or capture area argument, such as those based on the size distribution of Voronoi polyhedra around individual islands. ${ }^{11,15}$ The common feature of these equations is that the calculation of each capture number involves the solution of a diffusion equation, at the given densities $\left(n_{1}, n_{i}, n_{x}\right)$, which also contains the various capture numbers $\sigma_{1}, \sigma_{i}$, and $\sigma_{x}$ in the argument of the solution. These capture number equations can then be solved self-consistently as functions of $n_{1}, n_{i}$, and $n_{x}$ by numerical iteration.

Given this intrinsic complexity, it is natural to look for the simplest formulation first. For 2D problems on a substrate a radial diffusion equation is constructed as a function of $r$, neglecting any azimuthal $(\phi)$ variables, and the flux into an individual island is calculated to obtain the capture numbers. This, of course, may limit the physics, and it is known, especially from FIM studies on individual crystalline substrates, ${ }^{3,4}$ that such $\phi$ dependencies do exist and can be strong. However, for the case of $\mathrm{Cu} / \mathrm{Cu}(111)$ to be considered in Sec. V, azimuthal isotropy is a very good first approximation, as shown by isotropic nearest-neighbor distance histograms. $^{7}$

There is some possibility of confusion in the notation: $\theta$ cannot be used as an angle here, because it denotes the dose. When evaporation is unimportant, $\theta$ and $Z$ are often used interchangeably, as both are measured in monolayers (ML). But here we maintain the distinction, because the difference $(\theta-Z)$ can be important; for example, for 2D ML-thick islands and $i=1$, it represents the coverage of the substrate by adatoms. For 3D islands of a given shape the relationship is specific, but more complicated. ${ }^{10}$ We also have written $D_{1}$ for the adatom diffusion coefficient, in contrast to the more usual $D$; this is because we wish to define more than one type of diffusion coefficient.

The Bessel function form of the capture numbers for the diffusion-limited case has been well known since the 1970s, but has not been uniformly applied in subsequent papers. ${ }^{16,17}$ As a result it has sometimes been assumed that concern over self-consistency began in 1994 with Ref. 13; this topic has been carefully evaluated more recently in Ref. 16, which has clarified the record and made many useful observations and comparisons. As shown in Appendix A, the formulation in Ref. 13 for $i=1$ is the same as the uniform depletion approximation given in Ref. 10, where the contribution of the nucleation event itself to growth was neglected as being numerically unimportant. Thus the early and later rate equation formulations are in practice identical, and treatments based on Eq. (1) above can be more general.

What is particularly new in Ref. 13, and in many subsequent papers, is the comparison with KMC simulations ${ }^{15,16}$ and more recently with level set methods, ${ }^{18}$ especially for cluster size distributions $n_{s}(s)$ and spatial distributions. While the rate equations used in CNT yield essentially perfect agreement for average quantities such as $n_{1}$ and $n_{x}$, it is 
by now well known that they fail to account adequately for size and spatial distributions, because of the statistical nature of nucleation, and the fact that cluster growth depends on the local environment, i.e., on adatom-cluster correlations. There is also an intrinsic time dependence of the capture numbers themselves, dependent on initial spatial distributions. These features are the subject of ongoing work by several authors; ${ }^{15,18}$ the time dependence is not addressed in the first sections of the present paper, but is discussed in Sec. IV-VI, largely in relation to specific results for $\mathrm{Cu} / \mathrm{Cu}(111)$.

\section{DIFFUSION- VERSUS ATTACHMENT-LIMITED KINETICS}

Although the distinction between diffusion-limited and attachment-limited kinetics is generally well known in materials science, ${ }^{5}$ there have not been many publications in the subfield of epitaxial crystal growth, and the situation is rather confused. Thus, for example, it was concluded from a detailed first-principles theoretical study ${ }^{9}$ that repulsive interactions between adatoms rendered nucleation theory "inapplicable." As shown below, this conclusion is too strong, and should be replaced by the weaker statement, "a theory based on diffusion-limited kinetics alone may be inapplicable to systems where the repulsive interactions are of the same order of magnitude as the diffusion barrier". Attachmentlimited kinetics has only been explored with rate equations to the authors' knowledge in one paper, ${ }^{19}$ and the formulation and conclusions are reexamined below. In particular, it is found that the problem can be formulated more generally, and that there are two subcases leading to rather similar results. Attachment-limited kinetics has been addressed more recently via $\mathrm{KMC}$ simulations ${ }^{8,9,20}$ and one aim here is to compare the two approaches. As the present paper was finalized, another paper ${ }^{21}$ was published with similar interests to our own. Some brief comments are made in Sec. IV.

The first case is that there is an additional attachment barrier, of height $E_{B}$, at the interface of the growing island, as illustrated schematically in Fig. 1(a). This case was treated explicitly but not very generally in Ref. 19 , and led to a change in scaling law for the nucleation density in the steady-state regime. The scaling law derived in Ref. 19 is confirmed here in Appendix B, using a simpler argument, and preserving all the preexponential terms in the lowcoverage regime. This case constitutes a modification to the capture number $\sigma_{k}$ (i.e., $k=1, i, s$, or $x$, leading to $\sigma_{x}$ for the average-sized cluster) by an attachment-barrier capture number $\sigma_{B}$, which adds inversely as

$$
\sigma_{k}^{-1}=\sigma_{D}^{-1}+\sigma_{B}^{-1},
$$

where $\sigma_{D}$ is the diffusion-limited capture number. The capture number $\sigma_{D}$ is just the $\sigma_{k}$ given in Eq. (A2) in the uniform depletion approximation. These capture numbers add inversely because the diffusion flux across the barrier is conserved. In Ref. 19 the steady-state formula is derived in the low-coverage limit of the lattice approximation for $\sigma_{k}$, but Eq. (5) above shows that the result holds more generally. In this formulation, the extra barrier energy $E_{B}$ is incorporated
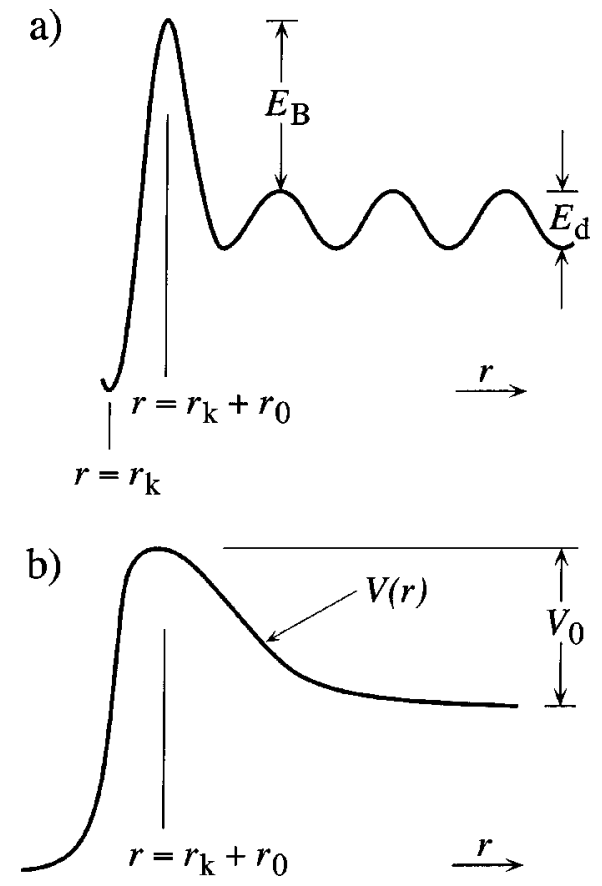

FIG. 1. Schematic energy-position diagrams of (a) diffusion over a uniform terrace with energy $E_{d}$, where the last step to join a $k$ cluster, radius $r_{k}$, has an additional barrier $E_{B}$; (b) energy landscape due to a long-range repulsive interaction, whose maximum is at $r=r_{k}+r_{0}$, with the value of $V(r)=V$ at large $r$; the important energy difference indicated is $V_{0}=V\left(r_{k}+r_{0}\right)-V$. Here the radial scale is presumed larger than in (a), and individual diffusion steps are not indicated.

into $\sigma_{B}$, rather than into a modified diffusion coefficient as in Ref. 19. The details are spelled out in Appendix B.

The specific case of complete condensation with $i=1$ and 2D islands is illustrated in Fig. 2 for two representative $\left(D_{1} / F\right)$ values. Each plot shows the capture numbers $\sigma_{x}$ and $\sigma_{i}$, as a function of dose, for both the diffusion-limited case (no barrier) and for three values of the barrier parameter $B$ $=2 \pi \exp \left(-\beta E_{B}\right)$. Note principally that as the value of $B$ is reduced, the capture numbers are reduced and become less dependent on dose, becoming dominated by the barrier capture number $\sigma_{B}$ in Eq. (5). In addition, Fig. 2 shows more subtle changes of value and shape, which are dependent on the $\left(D_{1} / F\right)$ values chosen. More detailed comments on these points are made later in the text, in the figure caption, and in Appendix B.

The second case arises when the individual adatoms and/or clusters have repulsive potential-energy fields $V(r)$ around them, with a range exceeding one lattice distance, as illustrated schematically in Fig. 1(b), where the diffusion barriers are not shown. Here it is primarily the change in energy landscape that is crucial in reducing $n_{1}(r)$ in the neighborhood of other adatoms and clusters, though this could also influence the adatom diffusion constant $D_{1}$, which can then depend on $r$. For this case, a different starting point is needed, as spelled out in Sec. IV. As shown below, the quantity $V_{0}=V\left(r_{k}+r_{0}\right)-V$, where the maximum is at $r=r_{k}+r_{0}$ and $V$ is the value at large $r$, plays a role similar to $E_{B}$ in the first case, if for different reasons. 

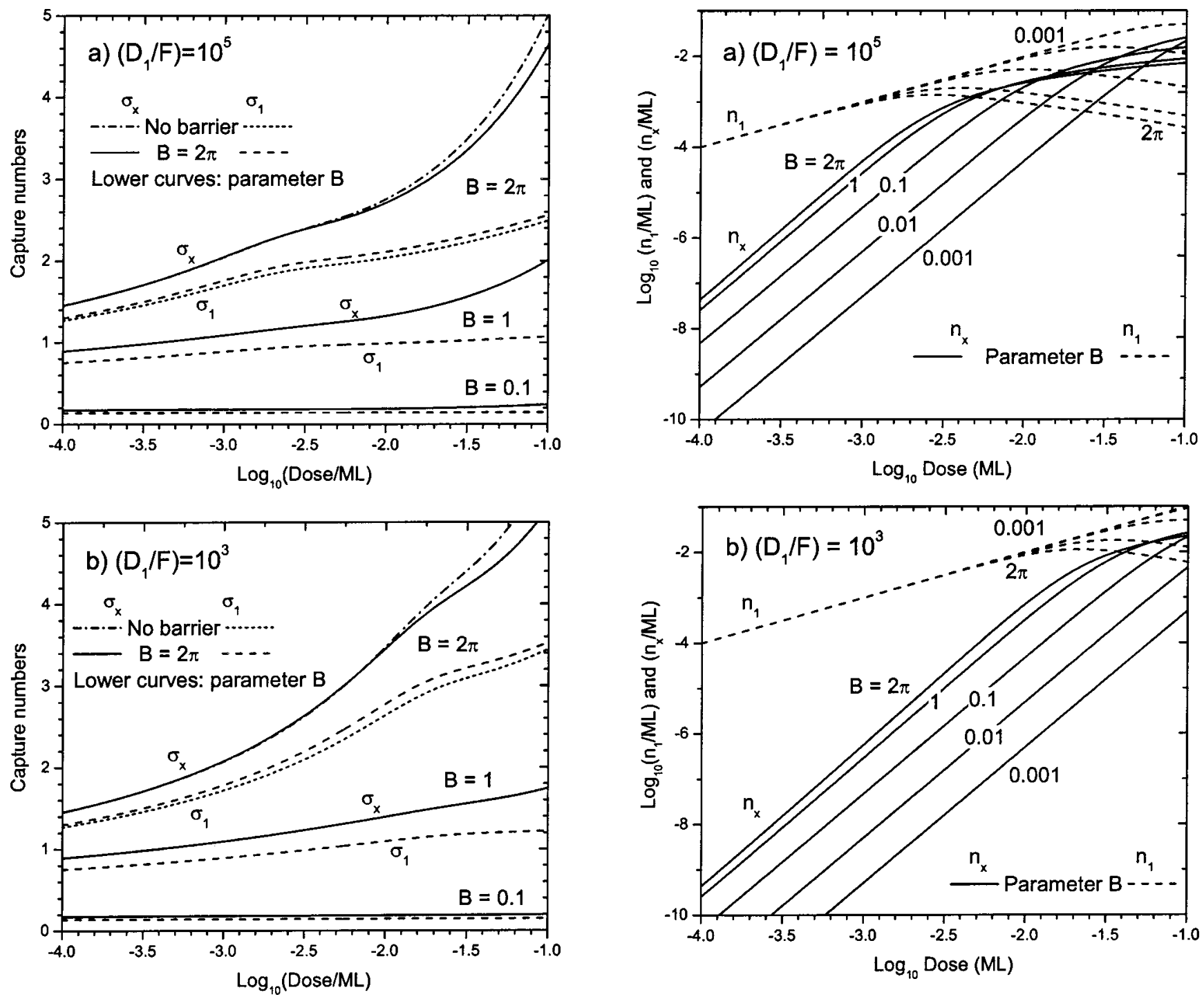

FIG. 2. Capture numbers $\sigma_{x}$ (full lines) and $\sigma_{i}$ (dashed lines, for $i=1$ ) as a function of dose [i.e., $\left.\log _{10}(\theta)\right]$, for three values of the barrier parameter $B=2 \pi \exp \left(-\beta E_{B}\right)=2 \pi, 1$, and 0.1 , for (a) $\left(D_{1} / F\right)=10^{5}$ and (b) $\left(D_{1} / F\right)=10^{3}$. See text for discussion and Appendix B for detailed conditions. For the capture numbers with no barrier, the curves are as indicated, and the correspondence with the case $B=2 \pi$ is discussed in Appendix B.

However, in both cases, an important point, apparently missed in Ref. 19, is that steady-state nucleation can be considerably delayed. This occurs because, with any or all of the capture numbers reduced by factors such as $\exp \left(-\beta E_{B}\right)$ or $\exp \left(-\beta V_{0}\right)$, the capture and/or nucleation times are increased by $\exp \left(+\beta E_{B}\right)$ or $\exp \left(+\beta V_{0}\right)$ to some power. This can be seen by inspection of Eqs. (1) and (2) above. The transient regime $n_{1}=F(1-Z) t=\theta(1-Z)$ ends only when the sum of the later terms balances this term, i.e., becomes equal to $\theta(1-Z)$ in Eq. (1) or $(1-Z)$ in Eq. (2). Thus if the capture numbers ( $\sigma_{1}$ and $\sigma_{x}$ in the $i=1$ case) are exponentially small, the transient regime can approach $1 \mathrm{ML}$. Similarly, for larger critical sizes $(i>1)$, if the nucleation rate [Eqs. (3) or (4)] becomes exponentially small via a reduction

FIG. 3. Log-log plots of $n_{1}$ and $n_{x}$ as a function of dose $\theta$, for the same two values of $\left(D_{1} / F\right)$ as in Fig. 2: (a) $\left(D_{1} / F\right)=10^{5}$ and (b) $\left(D_{1} / F\right)=10^{3}$, with a wider range of $B$ values as indicated. Note the increased importance of the transient regime for lower values of $B$, where $n_{1}=\theta$, and $n_{x}$ is roughly proportional to $\theta^{3}$ for $i=1$. See text for discussion and Appendices $\mathrm{B}$ and $\mathrm{C}$ for detailed conditions.

in $\sigma_{i}$, this regime, where all the deposit is in the form of monomers and subcritical clusters, becomes greatly lengthened.

We therefore need to explore how the nucleation density depends on dose in the transient regime in general, and in the various limiting cases; the details are given in Appendix C. The delayed onset of the steady-state regime with increasing $E_{B}$ causes most of the shape changes in $\sigma_{x}$ and $\sigma_{i}$ as a function of dose $\theta$, shown in Fig. 2 .

The longer transient regime is illustrated directly in the plots of $n_{1}$ and $n_{x}$ as a function of $\theta$ in Fig. 3, for the same two values of $\left(D_{1} / F\right)$, and a wider range of $B$ $=2 \pi \exp \left(-\beta E_{B}\right)$ values. It is seen that the transient regime (i.e., before the $n_{1}$ maximum, where $d n_{1} / d \theta=0$ ) can be dominant for quite modest values of $B$, especially at lower values of $\left(D_{1} / F\right)$. In Fig. 3(a) for $\left(D_{1} / F\right)=10^{5}$, the tran- 
sient regime extends up to $\theta=0.01 \mathrm{ML}$ for $B<0.1$; in Fig. 3 (b), for $\left(D_{1} / F\right)=10^{3}$, the corresponding limit for $\theta$ $=0.1 \mathrm{ML}$ is $B<0.01$. This trend has been followed both to higher $\left(D_{1} / F=10^{7}\right)$ and particularly to lower $\left(D_{1} / F=10\right)$ values, where it is well known that the transient regime is extensive even without barriers. ${ }^{16,26}$ Values of $\theta \gg 0.1 \mathrm{ML}$ are unrealistic in the simplest model, as we would need to consider coalescence and second layer formation in detail, neither of which forms the main point of this paper.

However, low values of $B$ are perfectly realistic: such values imply that a very high density of adatoms can be maintained in a long term, but metastable, state. In that case, the approximation that $\theta$ and $Z$ are interchangeable fails drastically; then steady-state formulas for the densities $n_{1}$ and $n_{x}$, and also for the capture numbers $\sigma_{1}$ and $\sigma_{x}$, are inappropriate. Such effects are needed for the evaluation of data taken at suitably low temperatures with strong enough attachment barriers. ${ }^{6,7}$ Once formulated, such a treatment is also suitable to follow annealing, including the (irreversible) formation of clusters when the temperature is high enough for the attachment barrier(s) to be surmounted.

\section{CAPTURE NUMBERS WITH LONG-RANGE REPULSIVE INTERACTIONS}

Recent STM work at low temperature ${ }^{6,7}$ has demonstrated the existence of oscillatory long-range interactions between individual adatoms on close-packed metal surfaces. This interaction has been explained by Friedel oscillations in the surface-state electrons, since it shows the characteristic asymptotic $E(r)=-A \sin \left(2 k_{F} r+2 \delta\right) / r^{2}$ dependence. ${ }^{22}$ Here, $A$ is the amplitude, $r$ the radial distance, $k_{F}$ the surface-state Fermi wave vector, and $\delta$ the scattering phase of the surfacestate electrons at the adsorbate. This asymptotic form of $E(r)$ is, when focusing on pair interactions, found to be valid down to $r=1 \mathrm{~nm}$ for $\mathrm{Cu}$ and $\mathrm{Co} / \mathrm{Cu}(111){ }^{7}$ While this oscillatory long-range interaction never exceeds $2 \mathrm{meV}$, there is a short-range repulsion for $r<0.7 \mathrm{~nm}$, which has been estimated to have a maximum of $13 \leqslant E_{\max } \leqslant 19 \mathrm{meV}$, localized at $r=1$ to 1.5 nearest-neighbor distances. ${ }^{7}$ We return to this system in Sec. V.

To describe the effect of such interactions on capture numbers, we need to start from a general position, which allows for an arbitrary, but in our case, radial-symmetric, potential-energy landscape $V(r)$ around each adatom and cluster. These interactions are mediated by electrons in the surface states of the substrate, and at very short range presumably also by elastic and direct interactions. These interactions are attached to the individual (moving) adatoms, so they are different in kind from those due to substrate defects. Indeed, due to the repulsion, their effect is just the opposite; they act to suppress nucleation rather than enhance it.

We are interested in solutions of a diffusion equation for the adatom concentration $n_{1}(r)$, when there are sources and sinks at various positions. The steady-state solution with $V(r)=0$ is typically known. ${ }^{1,2,10}$ The simplest case to think about is when we have a loss term governed by a characteristic lifetime $\tau$. The governing partial differential equation (PDE) is then

$$
\partial n_{1}(r) / \partial t=G-n_{1}(r) / \tau-\nabla . \mathbf{j}(r),
$$

where $\mathbf{j}(r)$ is the diffusive flux at radius $r$. The source term $G$ is equal to $F$ during deposition and typically zero during annealing. All variables in Eq. (6) are, in principle, functions of $r$ and $t$. The flux term $\mathbf{j}(r)$ is defined as $-D \nabla n_{1}(\mathbf{r})$, where $D$ is the chemical diffusion constant. Thus the last term in Eq. (6) is equal to $D \nabla^{2} n_{1}(r)$ if $D$ is indeed constant, independent of position and time.

But with nonzero $V(r)$ this starting point is not appropriate. There is much and somewhat confusing literature on this topic. We need to evaluate the response to concentration $[\nabla c(r)]$ and potential $[\nabla V(r)]$ gradients, via consideration of phenomenological transport coefficients, which leads to a more general definition of $\mathbf{j}(\mathbf{r})$ in terms of $\nabla \mu(\mathbf{r})$. This definition can be couched in terms of either $D$ or $D^{*}$, the tracer diffusion coefficient. It is acknowledged ${ }^{23}$ that it may be difficult to calculate either diffusion coefficient accurately, but that under certain reasonable conditions, ${ }^{24}\left(D / D^{*}\right)$ $=\beta\{\delta \mu / \delta[\ln (c)]\}$. The simplest expression for $\mathbf{j}(\mathbf{r})$, using this ratio, is

$$
\mathbf{j}(r)=-\left(D^{*} c\right) \beta \nabla \mu
$$

We now need the expression for $\mu(r)$ for a nonideal adsorbed gas, which is $\mu(r)=\mu_{0}+V(r)+\beta^{-1} \ln (\gamma c)$, where $\gamma$ is the activity. For this form of $\mu(r)$, the original definition of $\mathbf{j}(r)$ can be written as ${ }^{23,24}$

$$
\mathbf{j}(r)=-(D c)\{1+\delta \ln (\gamma) / \delta[\ln (c)]\}^{-1} \beta \nabla \mu ;
$$

the term $\{1+\delta \ln (\gamma) / \delta[\ln (c)]\}$ is known as the thermodynamic factor. This form $(7 \mathrm{~b})$ generalizes to include nonzero $V(r)$, but in any case these two expressions for $\mathbf{j}(r)$ are consistent, since for the assumed form of $\mu(r)$, $\delta \mu / \delta[\ln (c)]=\beta^{-1}\{1+\delta \ln (\gamma) / \delta[\ln (c)]\}$.

To calculate capture numbers in our mean-field model, we need the expression for $\mu(r)$ in the neighborhood of the adatom or cluster under consideration (at $\mathbf{r}=0$ ), in the presence of all the other adatoms and clusters. Thus the average, spatial independent $\mu$ can be subtracted off to give $\mu(r)$ $-\mu=V(r)-V+\beta^{-1}\{\ln [\gamma(r) c(r) /(\gamma c)]\}$. Here $V$ is the potential due to all the other adatoms and clusters at the mean density, so the large- $r$ limit of $V(r)=V$; the logarithmic concentration-dependent term depends on the ratio of the local value of $\left(\gamma_{c}\right)$ to its mean value. Pooling the above expressions (7), and using $c=n_{1}(r)$, we find that

$$
\mathbf{j}(r)=-D \nabla n_{1}(r)-\left[n_{1}(r) D^{*}\right] \beta \nabla V(r) .
$$

The derivative of $\gamma(r)$ is needed to obtain $D$ in the first term in Eqs. (8) from (7a), but $D^{*}$ remains in the second term. ${ }^{24}$ Moreover, if we assumed $\gamma(r)=\gamma$ in the model, the thermodynamic factor would be unity, so that $D$ and $D^{*}$ would be the same, but the following treatment is kept general for as long as possible.

$$
\begin{aligned}
& \text { Using Eq. (8), Eq. (6) becomes } \\
& \qquad \begin{aligned}
\partial n_{1}(r) / \partial t= & G-n_{1}(r) / \tau+\nabla \cdot\left[D \nabla n_{1}(r)\right] \\
& +\nabla \cdot\left\{\left[n_{1}(r) D^{*}\right] \beta \nabla V(r)\right\}
\end{aligned}
\end{aligned}
$$


Equation (9) is to be solved in various approximations. Note that although the $r$ dependence is written out explicitly for $n_{1}$ and $V$ and there is an implied time dependence, the "constants" $G, \tau, D$, and $D^{*}$ may also be functions of position (e.g., via concentration or a diffusion energy), or in the cases of $G, \tau$, of time (e.g., during deposition or annealing), without changing Eq. (9).

When $V(r) \neq 0$ and $D=D^{*}$, Eq. (9) can usefully be rewritten with $n_{1}=n \cdot \exp [-\beta V(r)]$, because then the last term in Eq. (9) is canceled by part of the expansion of the previous term. Now we are restricted to one diffusion coefficient, notionally the tracer diffusion coefficient. But real simplification is not possible unless $D^{*}$ is independent of position, in which case it is simplest to take $D^{*}=D_{1}$. It is, however, otherwise not necessary to assume low concentrations, or that $\gamma=1$. Equation (9) then yields a differential equation for $n$, rather than $n_{1}$, which can be reordered to give

$$
\begin{aligned}
\nabla^{2} n(r)-n(r) /\left(D_{1} \tau\right)= & {[\partial n(r) / \partial t] / D_{1}+\nabla n(r) \cdot \beta \nabla(r) } \\
& -\left(G / D_{1}\right) \cdot \exp [\beta V(r)],
\end{aligned}
$$

where in two dimensions, $\nabla^{2} n=\left(\partial^{2} n / \partial r^{2}+r^{-1} \partial n / \partial r\right)$. In passing, we note that essentially the same continuum problem in the presence of a radial symmetric potential has been studied independently very recently, ${ }^{21}$ via an atomistic formulation on a square lattice, yielding terms equivalent to Eqs. (9) and (10).

Equation (10) is clearly a form of Bessel equation, but with nonconstant coefficients, and nonzero terms on the right-hand side. Two limiting solutions are worth highlighting.

The first is the equilibrium case, where both $\nabla n(r)$ and $\nabla^{2} n=0$. Then in steady state,

$$
n(r) /\left(D_{1} \tau\right)=\left(G / D_{1}\right) \cdot \exp [\beta V(r)] .
$$

This equation is only consistent if $\tau=\tau_{0} \cdot \exp [-\beta V(r)]$, where $\tau_{0} \exp (-\beta V)$ is the constant value of $\tau$ at large $r$. Then during deposition, with $G=F, n_{1}(r)=F \tau_{0} \exp [-\beta V(r)]$ and $n_{1}(r \rightarrow \infty)=F \tau_{0} \exp (-\beta V)$. Thus, under these circumstances, $n_{1}$ simply reflects the Boltzmann distribution for the potential $V(r)$. This limit is easily visualized for the evaporation dominant case, where $\tau=\tau_{a}$. Then, at long times, $n_{1}$ $=F \tau_{a}$, with $\tau_{a}$ dependent on the adsorption energy $E_{a}$ as $\nu_{a}^{-1} \exp \left(\beta E_{a}\right)$, where $\nu_{a}$ is the appropriate frequency factor; ${ }^{14}$ it follows that with the repulsive energy $V(r)$, the expression is $\nu_{a}^{-1} \exp \left\{\beta\left[E_{a}-V(r)\right]\right\}$, which at large $r$ has the expected limit $\nu_{a}^{-1} \exp \left[\beta\left(E_{a}-V\right)\right]$, corresponding to a reduced adsorption energy. At finite coverage of the substrate by islands, $G=F(1-Z)$ in the above expressions; the argument generalizes to all times by considering Eqs. (10) and (1) together, giving the differential equation for $\left[n_{1}(r)\right.$ $\left.-n_{1}\right]$ in the same manner as Ref. 13.

The scaling of $n_{1}(r)$ with $\exp [-\beta V(r)]$ might be expected in the general case also, though this is certainly not obvious, and indeed $\tau$ may be considered to be an independent variable, to be determined iteratively alongside $n_{1}$ and $n_{x}$. At first, we consider $\left(D_{1} \tau\right)$ to be slowly varying spatially, so that the function needed to solve Eq. (10) has a similar form to Eq. (A1), namely,

$$
n(r)=G \tau_{0}\left[1-f(r) . K_{0}(X) / K_{0}\left(X_{k}\right)\right],
$$

where the function $f(r)$ is to be determined. Note that this equation still allows for time dependence, via the relation between $n_{1}$ and $G \tau_{0}$, and in the arguments $X$ and $X_{k}$, which are of the form $r /(D \tau)^{1 / 2}$ or equivalently $(r / \xi)$, see Appendix A. If we now demand that there is a perfect sink at the boundary of a $k$-sized island $r_{k}$ and convert back to $n_{1}(r)$, we find that the only solution of this form is

$$
n_{1}(r)=G \tau-G \tau_{0} \cdot \exp \left[-\beta V\left(r_{k}\right)\right] \cdot K_{0}(X) / K_{0}\left(X_{k}\right)
$$

or equivalently Eq. (12), with $f(r)=\exp \left\{\beta\left[V(r)-V\left(r_{k}\right)\right]\right\}$. From the definition of the capture number in terms of the flux $\left[\sigma(r) n_{1} D_{1}=-2 \pi r j(r)\right]$, taking the limit $r \rightarrow r_{k}$, we can show using Eq. (8) that the capture number corresponding to Eq. (13) is

$$
\begin{aligned}
\sigma_{k}= & \left\{2 \pi r_{k}\left[-\beta \nabla V\left(r_{k}\right)\right]+2 \pi X_{k} \cdot\left[K_{1}\left(X_{k}\right) / K_{0}\left(X_{k}\right)\right]\right\} \\
& \times \exp \left\{-\beta\left[V\left(r_{k}\right)-V\right]\right\} .
\end{aligned}
$$

Note that the capture number is reduced, relative to the standard diffusion expression (A2), by the Boltzmann factor $\exp \left\{-\beta\left[V\left(r_{k}\right)-V\right]\right\}$; this reduction can be substantial for high values of $\beta\left[V\left(r_{k}\right)-V\right]$. The first term in Eq. (14) is strictly zero if $V(r)$ has a maximum at $r=r_{k}$; however, there are contributions to $\sigma_{k}$ of order $2 \pi r_{k} \exp \left\{-\beta\left[V\left(r_{k}\right)-V\right]\right\}$ lurking if these conditions are not strictly fulfilled. In particular, this term has the form of an attachment-limited term $\sigma_{B}$ $=2 \pi r_{k} \exp \left(-\beta E_{B}\right)$, discussed in more detail in Sec. III and Appendix B, Eq. (B3). During the transient stage, before any spatial correlations have developed, this form of the capture numbers is dominant. Over time, correlations develop with the diffusion solution becoming dominant.

The next stage is to consider application of equations such as Eqs. (12)-(14) for specific forms of $V(r)$, and the effect on the nucleation density via the capture numbers $\sigma_{x}, \sigma_{1}$, and $\sigma_{i}$, i.e., via $\sigma_{k}$ in general. Some physical arguments are given in the text, and some comments on the mathematics are made in Appendix D. The main point is that Eq. (14) shows an exponentially reduced capture number over the usual diffusion solution. If $V(r)$ peaks at $r=r_{k}$, the solution is still typically diffusion limited. The radial distribution $n_{1}(r)$ evaluated according to Eq. (13) is shown in Fig. 4, for the specific case of a Lorentzian $V(r)$. Thus in Fig. 4(a) we show the case of $\left(D_{1} / F\right)=10^{5}$ and $\theta=0.01 \mathrm{ML}$, plotted as a function of $\left(r-r_{x}\right)$, with decreasing values of the parameter $B_{V}=2 \pi \cdot \exp \left\{-\beta\left[V\left(r_{x}\right)-V\right]\right\}$; note that the higher curves correspond to the lower values of $B_{V}$. It is clear that, although this is a diffusion solution, the form is very similar to that considered in the previous section for the attachment barrier with parameter $B$. But note that the reason is different; the main reason for the reduced capture number is thermodynamic, not kinetic, due to the reduction of $n_{1}\left(r_{k}\right)$ in the 

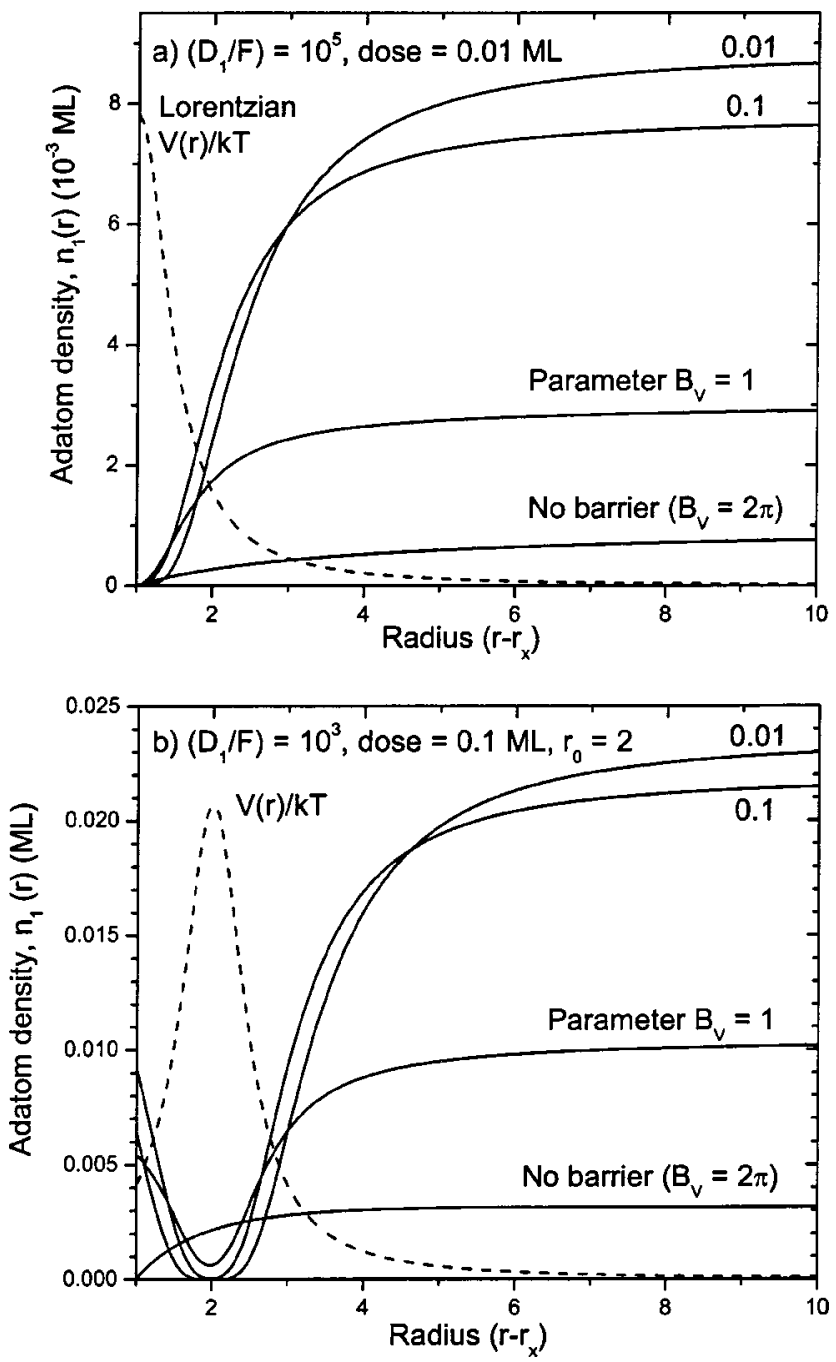

FIG. 4. Illustrative radial profiles for $n_{1}(r)$ and $[V(r)-V] / k T$ (dashed line) for four values of the potential parameter $B_{V}$ $=2 \pi \exp \left\{-\beta\left[V\left(r_{x}\right)-V\right]\right\}=2 \pi$ (no barrier), 1, 0.1, and 0.01: (a) $\left(D_{1} / F\right)=10^{5}, \theta=0.01 \mathrm{ML}$; (b) $\left(D_{1} / F\right)=10^{3}, \theta=0.1 \mathrm{ML}$, and $r_{0}=2$. Note that the higher curves correspond to the lower values of $B_{V}$, and that the profiles to the left of $r_{0}$ are modified by attachment. The potential illustrated schematically is Lorentzian with width $b=0.5$. See text for discussion and Appendix D for detailed conditions.

potential $\left[V\left(r_{k}\right)-V\right]$. There are no qualitative changes if we choose other (larger) doses at which to make this comparison.

However, if the maximum of $V(r)$ occurs at $r_{0}+r_{k}$, then Eq. (13) can go negative, yielding an unphysical result for $n_{1}(r)$ in the region $r-r_{k}<r_{0}$. This corresponds to a solution that is attachment limited at a larger radius; in other words, we have assumed a diffusion solution that cannot be maintained at a smaller radius. The real physical solution can be restored by considering a reduced diffusion gradient such that $n_{1}(r)$ is still positive at $r=r_{0}+r_{k}$. A single jump is typically all that is needed for the adatom to reach the sink at $r_{k}$, since the potential aids capture of adatoms for $r-r_{k}$ $<r_{0}$. Then Eq. (14) can be recalculated substituting this larger radius $r_{0}+r_{k}$ in the argument $X$ and in $\exp \{-\beta[V(r)$
$-V]\}$, as illustrated in Fig. 4(b) for $\left(D_{1} / F\right)=10^{3}, \theta$ $=0.1 \mathrm{ML}$, and $r_{0}=2$. Note that the solution of the rate equations does not need these radial plots, which are included for illustration; only the capture numbers $\sigma_{k}$ are needed, which are functions of $r_{0}$ and $r_{k}$, as set out in Appendix D. When the capture number involves both diffusion- and attachmentlimited terms they combine essentially as in Eq. (5). This point is illustrated using a single experimental example in the next section.

\section{NUCLEATION, ANNEALING, AND ENERGY BARRIERS ON CLOSE-PACKED METAL SURFACES}

In order to compare with experiment, we still need to program the equations described in this paper, and to take care of all the material and numerical constants with sufficient accuracy. We illustrate this here with a single example, $\mathrm{Cu} / \mathrm{Cu}(111)$, for which recent deposition and annealing data are available, ${ }^{6,7}$ and for which it is known we are dealing with complete condensation and small 2D islands. Two types of program have been developed in MATLAB ${ }^{\circledR} 5.3$ (student edition) to model:

(i) deposition processes, where output consists of (log$\log$ ) graphs of densities versus dose, and a file of the quantities $\sigma_{1}, \sigma_{x}, n_{1}$, and $n_{x}$, and the coverage of stable clusters $\left(Z=n_{x} w_{x}\right)$ at the final dose $\theta$;

(ii) annealing processes, using the output file from the deposition program as input. The output consists of similar files and graphs, as a function of annealing time, $V\left(r_{0}\right)$, and $r_{0}$.

The deposition program has been used to produce illustrative graphs of radial distributions $n_{1}(r)$, as, for example, Fig. 4. In order to use the minimum number of parameters, these runs have been restricted to $i=1$, for which the parameters during deposition are $\left(D_{1} / F\right)$ and either $B$ or $B_{V}$, and the independent variable is the dose $\theta$. In the annealing program $G=0$, and the calculation runs as a function of $\left(D_{1} t\right)^{0.5}$, anticipating the slowdown at longer times, and the main parameter is either $B$ or $B_{V}$. Minor parameters include $r_{0}$, and additional binding-energy parameters would be needed for both programs if $i>1$. The width parameter $b$ does not enter at the level of approximation represented by Eq. (14).

Material and deposition parameters known from experiment are then used to choose appropriate values of $F$ and $D_{1}$. The output of the comparison with experiment is the deduced value of $B$ or $B_{V}$, and hence the energy $E_{B}$ or $V_{0}$. In parallel with this comparison based on the rate equations, a KMC study has been done to test consistency between the two approaches.

The KMC simulations have been carried out on a hexagonal lattice of fcc sites. ${ }^{27}$ This is a good approximation for $\mathrm{Cu}$ monomer diffusion on the trigonal $\mathrm{Cu}(111)$ surface, since both experiment ${ }^{7}$ and theory ${ }^{8,20}$ find diffusion to occur between fcc sites only; i.e., the hcp site is as unstable as the bridge site. The simulations involve, apart from substrate temperature and deposition flux or annealing time and the fact that growth is irreversible $(i=1)$, the same three parameters as in Sec. III: the diffusion energy $E_{d}$ and attempt fre- 
quency $\nu$ for monomer diffusion, and the additional energy barrier $E_{B}$ for lateral attachment to an island or another monomer. The attempt frequency of the attachment process, which is the last jump towards an island or an adatom, is assumed also to be $\nu$; the total barrier for this process is $\left(E_{d}+E_{B}\right)$. The repulsive potential has its maximum at $1.5 a$ and is felt by atoms approaching an island or monomer from $2 a$ ( $a=$ nearest-neighbor distance). Atoms further away than this, or atoms diffusing away from, or parallel to, the island starting at distance $2 a$, diffuse with the unperturbed terrace barrier. Atoms deposited on top of an island, or a monomer, are allowed to descend the step and atoms deposited onto a site with a neighbor atom are stabilized there. To produce statistically meaningful results the simulations were performed several times on a large lattice $(1000 \times 1155$ sites $)$, each time with a different initializing value (seed) of the random number generator. ${ }^{28}$

In the limit of slow attachment, and sharp barriers, our two approaches should be identical. Specific features of low dose, low-temperature experiments, which may not be very important in general, have been incorporated into the rate equation treatment, as a result of comparison with the KMC simulations. These include the role of direct impingement on monomers during deposition, and of transient contributions to capture numbers during the initial stages of annealing.

Deposition of $\mathrm{Cu}$ atoms onto $\mathrm{Cu}(111)$ was performed in situ in a low-temperature STM, at $T=16.5 \mathrm{~K}$ at a flux $F$ $=5 \times 10^{-3} \mathrm{ML} / \mathrm{s}$ for a fraction of a second. ${ }^{7}$ The diffusion energy $E_{d}$ of $\mathrm{Cu}$ adatoms has been measured at somewhat higher temperatures as $40 \pm 1 \mathrm{meV}$, with a preexponential frequency factor $\nu=10^{12 \pm 0.5} \mathrm{~s}^{-1}, 7$ yielding $D_{1}$ $=0.156 \mathrm{ML} / \mathrm{s}$. These values give $\left(D_{1} / F\right)$ approximately equal to 30 during deposition. Figure 5 shows the evolution of $n_{1}$ and $n_{x}$ to the final dose $\theta=0.0014 \mathrm{ML}$. At such a low dose and value of $\left(D_{1} / F\right)$, both $n_{x}$ and $Z$ are very small, and almost all of the deposited material is in the form of monomers, and the few existing clusters are in the form of dimers.

The ratio $\left(n_{x} / n_{1}\right)$ is a sensitive test, not of $B$ or $B_{V}$, which only modify the already small diffusion terms, but of direct impingement on monomers. Direct attachment enters through the radius $r_{0}$, at which $V(r)$ has its maximum. In the rate equation solution behind Fig. 5, direct impingement adds to or creates a cluster, if the atoms fall within a radius $\left(r_{0}+r_{x}\right)$ or $\left(r_{0}+r_{1}\right)$, respectively, where we take $r_{1}=0.5$. A radius $r_{0}=1.5$ (measured from the origin) was suggested from the KMC simulations, and the ratio $\left(n_{x} / n_{1}\right)$ determined as $(4 \pm 2) \times 10^{-3}$; this point is the highest point plotted on Fig. 5. Given the uncertainties in experimental parameters, a value of $r_{0}$ in the rate equations (measured from the edge of the cluster) in the range $1-1.5$ predicts the ratio $\left(n_{x} / n_{1}\right)$, essentially independent of $B$ or $B_{V}$. Small values of these parameters serve merely to make diffusion even less important than it is already at low $\left(D_{1} / F\right)$ values.

Using the above values at the end of deposition, progress of $n_{1}, n_{x}$, and other quantities can be followed during annealing, as illustrated in Fig. 6(a) for $r_{0}=1.25$, chosen to agree precisely with the initial values in the KMC simulation data shown. The extent of cluster formation can be seen by the rise in $n_{x}$ that accompanies the fall in $n_{1}$ as a function of

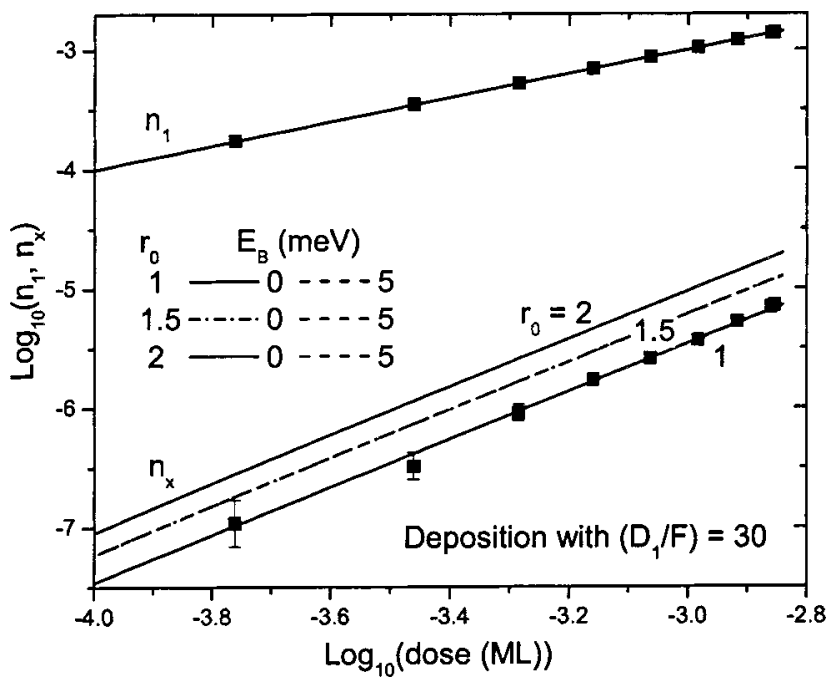

FIG. 5. Predicted $n_{x}(\theta)$ curves for deposition with $\left(D_{1} / F\right)$ $=30$, and reasonable $r_{0}$ values $1,1.5$, and 2 . Lines for $B_{V}=2 \pi$ (no barrier) and 0.187 , corresponding to a $5-\mathrm{meV}$ barrier at $16.5 \mathrm{~K}$ are plotted, but fall essentially on top of each other; higher barriers give negligible further changes. The data points are the result of a KMC simulation with $\mathrm{Cu} / \mathrm{Cu}(111)$ parameters $\left(E_{d}=40 \mathrm{meV}, \nu=1 \mathrm{THz}\right)$. Consistent with densities inferred from experiment, these simulations produced 357 dimers and $\sim 6.4 \times 10^{4}$ monomers at $\theta$ $=0.0014 \mathrm{ML}$. Atoms deposited on top of an island or monomer were allowed to descend and attach laterally to the island or monomer.

$\left(D_{1} t\right)^{0.5}$, with parameter $B_{V}$. Again, the absolute values of $n_{1}, n_{x}$, and especially the ratio $\left(n_{x} / n_{1}\right)$ are sensitive tests of the parameters in the rate equation model.

The steady-state mean-field capture numbers were initially used with this range of $B_{V}$ values, corresponding to 0 $<E_{B}<10 \mathrm{meV}$. As seen in Fig. 6(a), dashed curves, this leads us to underestimate the amount of annealing, for all $B_{V}$ values. The basic reason is clear: steady-state capture numbers are appropriate when spatial correlations are fully developed, which they are certainly not in the early stages of either deposition or annealing. In the "completely uncorrelated" limit, before a diffusion field has developed, the capture number is given by the pure attachment limit, derived in Appendix B [Eq. (B3)]. For the case where $r_{0}>1$ and a potential barrier $V_{0}$ we have used the form

$$
\sigma_{B}=\left(r_{k}+r_{0}\right) B_{V}=2 \pi\left(r_{k}+r_{0}\right) \exp \left(-\beta V_{0}\right) .
$$

The curves for $V_{0}=E_{B}=5$ and $10 \mathrm{meV}$ are in essential agreement with this simple formula over the whole range of annealing conditions shown in Fig. 6(a). By extension, they would also be in perfect agreement with Eq. (15) for all larger values of $V_{0}$ or $E_{B}$, but of course for larger energy values there is almost no annealing over the range of $\left(D_{1} t\right)^{0.5}$ shown.

At the other end of the scale, Eq. (15) gives far too much annealing when $V_{0}=E_{B}=0$, most obviously seen in Fig. 6(a) by the gross discrepancy in the prediction of $n_{1}$. Thus, for the lower values of $V_{0}$ or $E_{B}$, or for longer annealing times, an interpolation scheme is needed, which will take the value 

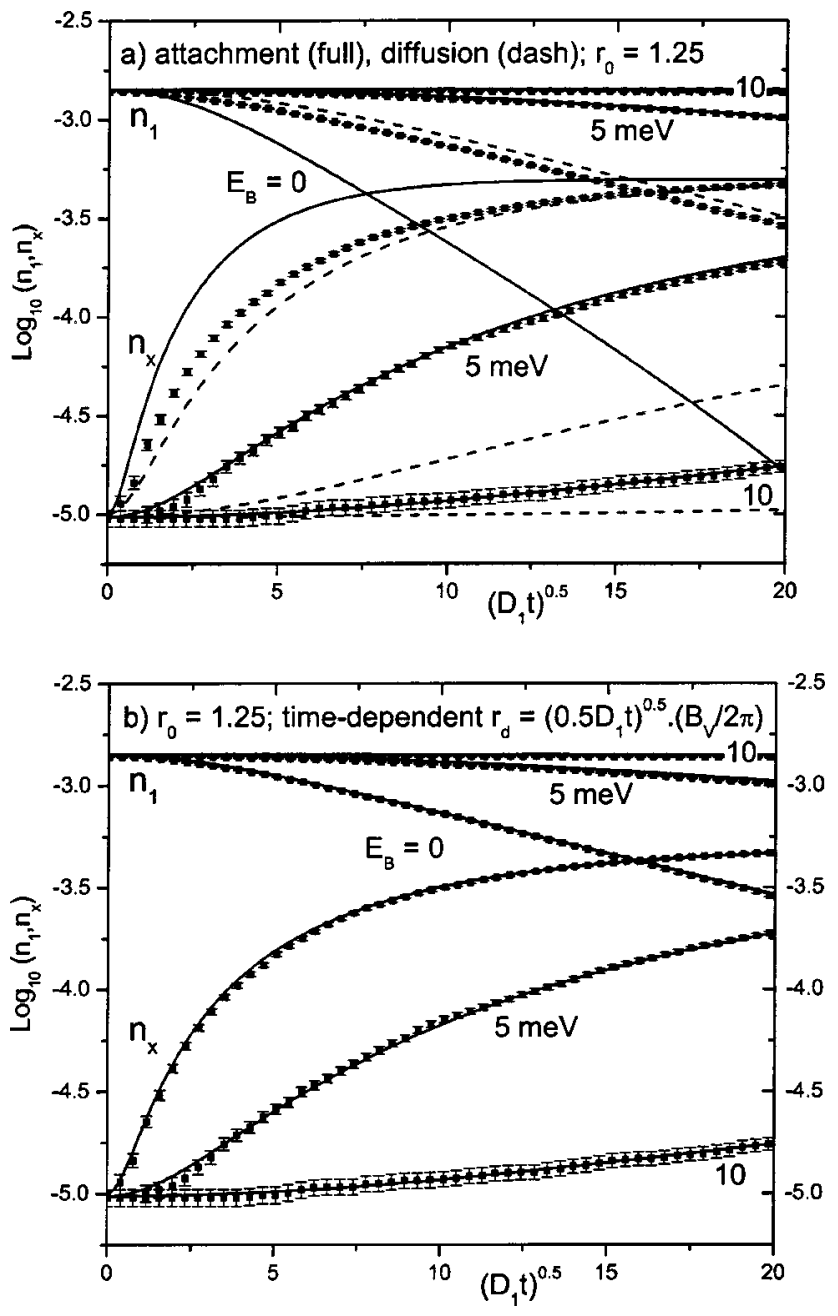

FIG. 6. Predicted $n_{1}$ and $n_{x}$ annealing curves as a function of $\left(D_{1} t\right)^{0.5}$, for $B_{V}$ values which correspond to annealing at $16.5 \mathrm{~K}$ with attachment barriers $E_{B}=0,5$, and $10 \mathrm{meV}$, compared to KMC simulations (squares with error bars): (a) capture numbers corresponding to attachment-limited solutions (full lines) and diffusion solutions (dashed lines); (b) capture numbers based on the timedependent interpolation scheme between attachment and diffusion solutions, showing essential agreement with the KMC simulations. The initial data file corresponds to the final values displayed in Fig. 5 , with $r_{0}=1.25$. See text for discussion of how these curves apply to STM experiments on $\mathrm{Cu} / \mathrm{Cu}(111)$.

of the capture number from an initial uncorrelated value to a final diffusive value over the correct range of $\left(D_{1} t\right)$. By examination of the coupled rate equations for annealing, analogous to Eqs. (2) and (4) for deposition, we can see that the independent variable is $\left(D_{1} t\right)$, and that the only other parameter is $B_{V}$, which enters via Eq. (15). The details are given in Appendix D.

A particular set of the resulting curves is shown in Fig. $6(\mathrm{~b})$, based on the formula

$$
\sigma_{k}=\left(\sigma_{B} f_{t}\right) f_{t}+\sigma_{k d}\left(1-f_{t}\right),
$$

where $\sigma_{k d}$ is the diffusive contribution given by Eq. (14), and $f_{t}$ is a transient factor, such that at $\left(D_{1} t\right)=0, f_{t}=1$, and as $\left(D_{1} t\right) \rightarrow \infty, f_{t} \rightarrow 0$. Short of a complete closed-form solu-

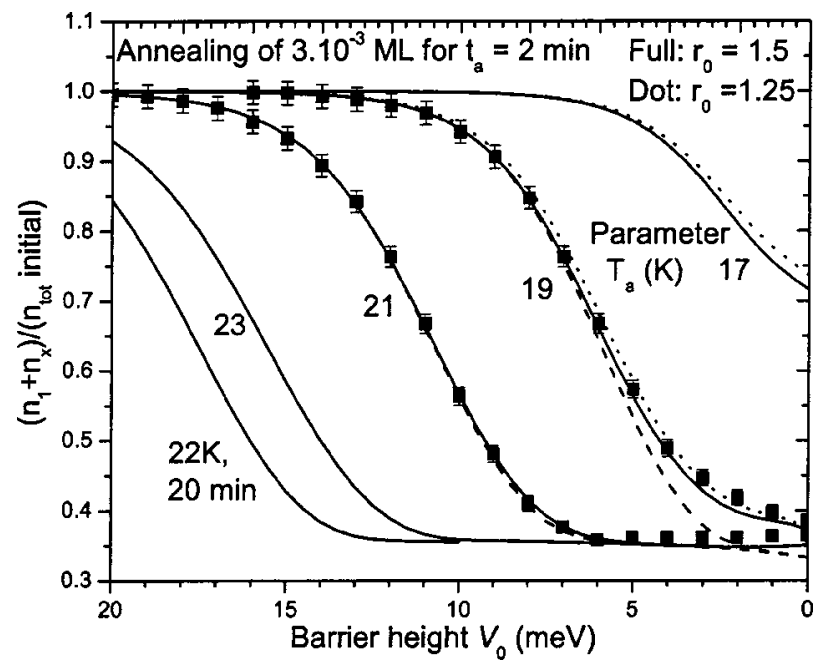

FIG. 7. Predicted annealing curves as a function of barrier height $V_{0}$, at temperatures $17<T_{a}<23 \mathrm{~K}$. Plotted is the ratio $\left(n_{1}\right.$ $\left.+n_{x}\right)$ after a 2-min anneal, divided by the initial value $n_{\text {tot }}=\left(n_{1}\right.$ $\left.+n_{x}\right)$ after deposition. The full curves use the time-dependent capture number expression for $r_{0}=1.5$, with the dotted curves having $r_{0}=1.25$ as in Fig. 6; the dashed curves for $T_{a}=19$ and $21 \mathrm{~K}$ correspond to attachment-limited capture numbers, which are a good approximation for all anneals initially. The curves for 19 and $21 \mathrm{~K}$ are also compared with the KMC simulations (squares with error bars). Additionally a curve for annealing at $22 \mathrm{~K}$ for $20 \mathrm{~min}$ is given. See text for discussion of how these curves apply to STM experiments on $\mathrm{Cu} / \mathrm{Cu}(111)$, and Appendix D for details of the algorithms used.

tion to time-dependent equations for the capture numbers, we have experimented with justifiable forms of $f_{t}$; our best fit to date is given in Fig. 6(b). The physical argument used is that the transient is due to capture from a diffusion zone around the adatom or cluster considered, whose radius $r_{d}$ increases with time as some function of $\left(D_{1} t\right)$ and $B_{V}$. The details are discussed in Appendix D.

In the corresponding annealing experiment for $\mathrm{Cu} / \mathrm{Cu}(111),{ }^{7}$ no further cluster formation was observed during annealing at $16.5 \mathrm{~K}$ for $20 \mathrm{~min}$. On the other hand, almost all the monomers formed clusters during annealing at $22 \mathrm{~K}$ for a comparable time. Thus we can put upper and lower bounds on the value of $B_{V}$ and hence bounds on the maximum value of $V(r)$, i.e., $V_{0}$. From Fig. 6, we can deduce, using $\left(D_{1} t\right)^{0.5}$ at $20 \mathrm{~min}=13.4$, that the lower bound to $V_{0}$ is around $10 \mathrm{meV}$. The upper bound can be estimated from Fig. 7. This figure shows the ratio $\left(n_{1}+n_{x}\right) /\left(n_{\text {tot }}\right)$, where $n_{\text {tot }}$ is the initial sum of $\left(n_{1}+n_{x}\right)$ after deposition at $16.5 \mathrm{~K}$, as a function of barrier height $V_{0}$, using the above interpolation scheme with the KMC simulations for $t$ $=120-\mathrm{s}$ anneals at $T=19$ and $21 \mathrm{~K}$. The rate equation ratios are given for $17,19,21$, and $23 \mathrm{~K}$, and for $22 \mathrm{~K}$ for $20 \mathrm{~min}$; the constants are the same as in Fig. 6, so the fit is a good test of the interpolation scheme proposed. Note that, according to the rate equation integration shown in Fig. 7, annealing at 22 $\mathrm{K}$ for $20 \mathrm{~min}$ is comparable to annealing at around $23.8 \mathrm{~K}$ for $2 \mathrm{~min}$; this could not be checked by KMC simulations because of the excessive amount of computer time needed. 
From the reported onset of dimer formation for $\theta$ $=0.003 \mathrm{ML}$ between 19 and $21 \mathrm{~K}^{7}$ we can infer that 9 $<V_{0}<14 \mathrm{meV}$ from Fig. 7. This result assumes that the onset of dimer formation corresponds to a $10 \%$ decrease in the total density $\left(n_{1}+n_{x}\right)$ after 2-min annealing. Since, however, time and coverage were not specified precisely in Ref. 7, we prefer to retain the estimate derived from the considerations in the previous paragraph. This maximum, $V\left(r_{0}\right)$, lies between 10 and $14 \mathrm{meV}$, and is not very sensitive to the exact choice of the radius $r_{0}$. This lack of sensitivity to $r_{0}$ arises because direct impingement terms are not active during annealing, and diffusion terms are modified only slightly by $r_{0}$. The energy values deduced are, however, sensitive to the choice of capture number expression, and do require the inclusion of transient effects in the capture numbers themselves, as discussed above.

\section{DISCUSSION AND CONCLUSIONS}

We have examined the form of capture numbers, and presented rate equations for deposition and annealing, including the effects of additional attachment barriers, both for extra diffusion barriers and for potential fields of longer range, in the neighborhood of adatoms and clusters. We have derived the form of the adatom and nucleation density as a function of material parameters in various subcases. We have shown that such barriers extend the transient regime in the early stages of deposition. Analytic expressions for these capture numbers predict cluster densities in essentially perfect agreement with kinetic Monte Carlo simulations during lowtemperature deposition. However, direct impingement, even onto individual adatoms, can be an important process, if diffusion capture is hindered by attachment barriers.

Transient effects on capture numbers in the presence of the repulsive potential $V(r)$ have been estimated for the case of $\mathrm{Cu} / \mathrm{Cu}(111)$. For the experimental doses and annealing times, they increase the capture numbers initially, and reduce in importance during annealing. A suitable interpolation scheme is proposed, which spans the transient regime. We have used this scheme to obtain lower and upper bounds to the maximum in the repulsive potential $10<V_{0}<14 \mathrm{meV}$, somewhat lower than a previous estimate of $13-19 \mathrm{meV}^{7} \mathrm{By}$ quoting relatively wide error bars, we have ensured that any remaining uncertainties in the capture numbers do not affect our quantitative conclusions about energies in the $\mathrm{Cu} /$ $\mathrm{Cu}$ (111) system. There may still be some further avenues to be explored, but these are probably best approached in the context of particular experimental results, since otherwise the number of underdetermined parameters can become too large.

\section{ACKNOWLEDGMENTS}

Discussions with A. V. Sobolev on differential equations, and a detailed reading of the paper by $\mathrm{C}$. Ratsch, were helpful and are much appreciated.

\section{APPENDIX A: DIFFUSION SOLUTIONS FOR CAPTURE NUMBERS}

The uniform depletion solution for the radial dependence $n_{1}(r)$ on a $2 \mathrm{D}$ substrate, with a perfect sink at $r=r_{k}$, is given by (Ref. 10, where $R=F$ )

$$
n_{1}(r)=F \tau(1-Z)\left[1-K_{0}(X) / K_{0}\left(X_{k}\right)\right] ;
$$

the corresponding capture number is obtained from the flux equation $\mathbf{J}=-D_{1} \nabla n_{1}(r)$ evaluated at $r=r_{k}$, as $\sigma_{k} D_{1} n_{1}$, giving

$$
\sigma_{k}=\left(2 \pi X_{k}\right) \cdot K_{1}\left(X_{k}\right) / K_{0}\left(X_{k}\right) .
$$

The arguments $X=r /\left(D_{1} \tau\right)^{1 / 2}$ and $X_{k}=r_{k} /\left(D_{1} \tau\right)^{1 / 2}$ of the Bessel functions $K_{0}$ and $K_{1}$ set the length scale where the adatom density is depleted in the vicinity of a $k$ cluster. The self-consistency condition arises because these arguments are themselves a function of (other) capture numbers.

For example, in the complete condensation case, where the dominant contribution to $\tau^{-1}$ is $\tau_{c}^{-1}=\sigma_{x} D_{1} n_{x}, X_{k}^{2}$ $=\sigma_{x} n_{x} r_{k}^{2}$. So if Eq. (A2) is evaluated for the average size cluster $\left(k=x, r_{k}=r_{x}\right)$ we can write $X_{x}^{2}=\sigma_{x} n_{x} r_{x}^{2}=\sigma_{x} Z / \pi$, and the capture number depends only on $Z$. Similarly, if we consider the capture by $k$ clusters in the presence of all the other clusters, $\sigma_{k}$ is only a function of $Z_{k} / Z=\left(r_{k} / r_{x}\right)^{2}$. These features have been illustrated in the capture number calculations of Ref. 10, Fig. 2, where the independent variable is $Z$. Note that in almost all subsequent papers, quantities are plotted as a function of dose $\theta$.

Bales and Chrzan $^{13}$ described the same phenomena in terms of a characteristic length scale $\xi$, so that the argument $X$ in Eq. (A1) is just $(r / \xi)$. The expression for $\sigma_{k}(k=s$ in Ref. 13) is Eq. (A2), and from the correspondence, $\xi^{2}$ $=D_{1} \tau$. These authors included all the terms relevant for complete condensation when $i=1$, including the two diffusive terms given here in the text in Eq. (2), and the small direct impingement term (atoms falling directly onto adatoms). Without this last term, $\xi^{-2}$ is given by

$$
\xi^{-2}=2 \sigma_{1} n_{1}+\sigma_{x} n_{x} .
$$

Thus Eq. (A3) can be incorporated into Eq. (A2), with the added complexity that $\sigma_{k}$ now depends implicitly on both $\sigma_{x}$ and $\sigma_{1}$, or in general $\sigma_{i}$. Equation (A3) is the same as the text Eq. (1), where the two terms $\tau_{n}^{-1}$ and $\tau_{c}^{-1}$ have been retained. These equations can thus be extended to include other processes, such as evaporation, and for general $i$ values, but if several processes are important simultaneously, simple scaling with material parameters will be lost; this is the price of completeness.

\section{APPENDIX B: STEADY-STATE EFFECTS OF ATTACHMENT BARRIERS}

\section{Introduction and summary}

Kande ${ }^{19}$ has given a discussion of the effect of island edge barriers, for $2 \mathrm{D}$ circular islands, with no reevaporation or coalescence, and a general value of the critical cluster size $i$. He showed that in steady-state conditions, the nucleation 
density scaling could be changed from the diffusion-limited value $i /(i+2)$ to the larger attachment-limited value $2 i /(i$ $+3)$. This exponent is confirmed here by a more transparent argument, which does not depend on the particular form of capture number he used, which was equivalent to the lattice approximation at low coverage. It is also shown here that when there is an attachment barrier, the capture numbers for diffusion $\sigma_{D}$ and for the barrier $\sigma_{B}$ combine inversely as in Eq. (5); this equation is valid in general, as it rests on the continuity of the diffusion flux across the barrier.

We start here from Eqs. (2) and (4), in the case where the nucleation term [e.g., $2 \sigma_{1} D_{1} n_{1}^{2}$ in Eq. (2)] and coalescence term $\left[U_{c}\right.$ in Eq. (4) $]$ are numerically negligible. In steadystate conditions, we then have from Eq. (2) $n_{1}=F \tau_{c}(1$ $-Z$ ), where $\tau_{c}^{-1}=\sigma_{x} D_{1} n_{x}$, as in Appendix A. Inserting this value of $n_{1}$ into Eq. (4) yields in the limit of low island coverage $(Z \ll 1)$,

$$
d n_{x} / d \theta=\left(\sigma_{i} C_{i}\right) \cdot\left(\sigma_{x} n_{x}\right)^{-(i+1)} \cdot\left(D_{1} / F\right)^{-i} \exp \left(\beta E_{i}\right) .
$$

We can integrate Eq. (B1) to obtain

$$
\begin{aligned}
& {\left[n_{x}^{(i+2)} /(i+2)\right]} \\
& \quad=\left(D_{1} / F\right)^{-i} \exp \left(\beta E_{i}\right) \cdot \int\left\{\left(\sigma_{i} C\right) \cdot \sigma_{x}^{-(i+1)} \cdot d \theta\right\} .
\end{aligned}
$$

From the form of Eq. (B2), we can see that the normal $n_{x}$ power-law scaling with $\left(F / D_{1}\right), i /(i+2)$ is obtained provided that the integral over the capture numbers in Eq. (B2) is well defined, and does not contain any material parameters. This is the case for complete condensation, where the capture numbers are slowly varying Bessel functions as outlined in Appendix A. A full discussion of various numerical possibilities was given in Ref. 16.

\section{Power-law modification by attachment barriers}

In the presence of an attachment energy barrier $E_{B}$, there are various ways that the above equations could be modified. We can change either $\sigma_{x}$ or $\sigma_{i}$ or both. In Ref. 19 new variables $S=D \exp \left(-\beta E_{B}\right)$ and $S^{*}=D \exp \left(-\beta E_{B}^{*}\right)$ were introduced to modify stable and critical cluster capture processes, respectively, but the definitions of $\sigma_{x}$ and $\sigma_{i}$ were left as before. That ansatz associates the barrier uniquely with diffusion in the mind of the reader; specific examples were given only for $E_{B}^{*}=E_{B}$. Here, we prefer to incorporate the barrier properties into the definition of the general capture number $\sigma_{k}$, as the different limits then arise naturally.

The diffusion limit $\sigma_{D}$ in the mean-field approximation is just $\sigma_{k}$ given in Eq. (A2). It remains to estimate the attachment limit $\sigma_{B}$. We can see qualitatively that when the attachment limit is needed near $r=r_{k}$, there will be little longrange diffusion field, and $n_{1}\left(r_{k}+1\right) \sim n_{1}$, the average value. ${ }^{25}$ The number of sites around the periphery of the cluster is of order $2 \pi\left(r_{k}+1\right)$; from these sites, adatoms can jump towards the cluster with probability per unit time $\left(\nu_{d} / 4\right) \exp \left[-\beta\left(E_{B}+E_{d}\right)\right]$. The rate at which adatoms join the cluster is by definition $\sigma_{B} D_{1} n_{1}$, so for circular clusters

$$
\sigma_{B}=2 \pi\left(r_{k}+1\right) \exp \left(-\beta E_{B}\right),
$$

where we have used the usual form, in ML units, $D_{1}$ $=\left(\nu_{d} / 4\right) \exp \left(-\beta E_{d}\right)$.

When both diffusion and attachment barriers are important, the adatom density is depressed part of the way toward the diffusion solution, but the flux across the interface region must be consistent with the interface concentration $n_{1}\left(r_{k}\right.$ $+1)$. Thus there are two expressions for the diffusion flux. For $r \geqslant\left(r_{k}+1\right)$, we have the diffusion solution analogous to Eq. (A1),

$$
n_{1}(r)=n_{1}-\left\{\left[n_{1}-n_{1}\left(r_{k}+1\right)\right] . K_{0}(X) / K_{0}\left(X_{k}\right)\right\},
$$

with $n_{1}=F \tau(1-Z)$. The effective diffusive capture number is, analogous to Eq. (A2),

$$
\sigma_{D e}=\left(1-f_{B}\right) \cdot\left(2 \pi X_{k 1}\right) \cdot K_{1}\left(X_{k 1}\right) / K_{0}\left(X_{k l}\right)=\left(1-f_{B}\right) \sigma_{D},
$$

where the factor $f_{B}=\left[n_{1}\left(r_{k}+1\right)\right] / n_{1}$.

However, there is also the solution derived from Eq. (B3). Here the effective capture number is $\sigma_{B}\left[n_{1}\left(r_{k}+1\right)\right] / n_{1}$ $=\sigma_{B} f_{B}$. Since both this quantity and Eq. (B5) constitute the same $\sigma_{k}$, we can determine the factors $f_{B}=\sigma_{D} /\left(\sigma_{D}+\sigma_{B}\right)$ and $\sigma_{k}=\sigma_{B} \sigma_{D} /\left(\sigma_{D}+\sigma_{B}\right)$. This last expression shows that the diffusive and barrier capture numbers add inversely as $\sigma_{k}^{-1}=\sigma_{D}^{-1}+\sigma_{B}^{-1}$, which is given in the text as Eq. (5).

Note, as a detail, that the argument $X_{k 1}$ of Eq. (B5) should be evaluated at $r=\left(r_{k}+1\right)$, not at $r_{k}$, as in Eq. (A2). In Fig. 2 , we have experimented with this detail, and found that the calculation is a bit sensitive to the exact choice of boundary condition; overinterpretation of a continuum model is of course suspect for clusters of atomic size. Thus the line for $B=2 \pi$ (i.e., $E_{B}=0$ ) should fall on top of the no-barrier line if all boundary conditions were exact. Figure 2 shows the case where the argument is evaluated $r=\left(r_{k}+0.5\right)$, but a range of constants give very similar results. ${ }^{25}$ The divergence between the "no-barrier" and $B=2 \pi$ curves at high dose is due to the need to change other boundary conditions, including modifying direct impingement terms. Since there are several constants that could be changed marginally, with no guarantee of self-consistency, we have left Fig. 2 unchanged. Such changes have negligible effects on Fig. 3, where the lines for $B=2 \pi$ and the "no-barrier" essentially coincide, so only one is shown.

We are now in a position to see that Kandel's limit ${ }^{19}$ (II) arises when $\sigma_{B} \ll \sigma_{D}$, so that $\sigma_{k}=\sigma_{B}$. If we use expression (B3) for both $\sigma_{i}$ and $\sigma_{x}$ in Eq. (B2), we have

$$
\sigma_{i} \sigma_{x}^{-(i+1)}=\left(r_{i}+1\right) \cdot\left(r_{x}+1\right)^{-(i+1)} \cdot\left[2 \pi \exp \left(\beta E_{B}\right)\right]^{i} .
$$

This is not yet in the right form, but note the term in $r_{x}$, which is coverage dependent. In complete condensation at island coverage, $Z=\pi n_{x} r_{x}^{2}$. If we neglect both the difference between $Z$ and $\theta$ and the extra terms of order 1, and assume $r_{i} \sim i^{1 / 2}$ in Eq. (B6), then we have 


$$
\sigma_{i} \sigma_{x}^{-(i+1)}=\left(i^{1 / 2}+1\right)\left(\theta / \pi n_{x}\right)^{-(i+1) / 2} \cdot\left[2 \pi \exp \left(\beta E_{B}\right)\right]^{i} .
$$

By transferring the term in $n_{x}^{-(i+1) / 2}$ to the left-hand side of Eq. (B1) and integrating, we obtain

$$
\begin{aligned}
\left\{n_{x}^{(i+3) / 2} /[(i+3) / 2]\right\}= & \left(D_{1} / F\right)^{-i} \exp \left[\beta\left(E_{i}+i E_{B}\right)\right] \cdot \int\left\{\left(i^{1 / 2}\right.\right. \\
& \left.+1) C_{i} \cdot 2 \pi^{i} \cdot(\theta / \pi)^{-(i+1) / 2} d \theta\right\} .
\end{aligned}
$$

Equation (B8) shows the $2 i /(i+3)$ power-law scaling with $\left(F / D_{1}\right)$ for the nucleation density, confirming Kandel's limit II for steady-state attachment-limited kinetics, while retaining all the numerical constants. It is also clear from Eq. (B7) that the power law is due to $\sigma_{x}$, not to $\sigma_{i}$; if there were no barrier term in $\sigma_{i}$, Eq. (B8) would remain the same except for the slightly modified Arrhenius dependence $\exp \left\{2 \beta\left[E_{i}+i E_{d}+(i+1) E_{B}\right] /(i+3)\right\}$. If there were a barrier term in $\sigma_{i}$ but not in $\sigma_{x}$, then the power-law scaling would be "normal," i.e., as in Eq. (B2), but with Arrhenius dependence $\exp \left[\beta\left(E_{i}+i E_{d}-E_{B}\right) /(i+2)\right]$. Note that in this case, $E_{B}$ reduces the nucleation density somewhat, whereas in Eq. (B8) $E_{B}$ increases it substantially.

\section{Conclusion}

Although we have shown that Kandel's steady-state formula is functionally correct, the formula may be of limited usefulness. The reduced capture numbers due to barriers lead to increased importance of the transient regime, for which his formula is not applicable. The $2 i /(i+3)$ power-law results from the equality of $Z$ and $\theta$ in Eq. (B7) which is only valid for large $2 \mathrm{D}$ islands and small values of $n_{1}$. Both of these conditions are violated in the transient regime.

\section{APPENDIX C: TRANSIENT NUCLEATION}

The nucleation rate in the transient regime is given from Eqs. (3) or (4), neglecting the coalescence term, as

$$
d n_{x} / d \theta=\sigma_{i}\left(D_{1} / F\right) C_{i} \theta^{(i+1)} \exp \left(\beta E_{i}\right) .
$$

Within the transient regime $n_{1}=F t=\theta$, so the nucleation density is given as

$$
\left[n_{x}\right]=\left(D_{1} / F\right) \exp \left(\beta E_{i}\right) \cdot \int \sigma_{i} C_{i} \theta^{(i+1)} d \theta .
$$

The end of the transient regime occurs at time $\tau$ given in Eq. (1), so the details depend on the condensation regime. For complete condensation, $\tau$ is $\tau_{c}$ or, equivalently, $\theta_{c}$. At this stage,

$$
n_{x}\left(\tau_{c}\right)=\left(D_{1} / F\right) \exp \left(\beta E_{i}\right) \cdot \int \sigma_{i} C_{i} \theta^{(i+1)} d \theta,
$$

where the upper limit is $\theta_{c}=F \tau_{c}=\left(F / D_{1}\right)\left(\sigma_{x} n_{x}\right)^{-1}$, and the lower limit is zero. The power-law scaling for $n_{x}\left(\tau_{c}\right)$ depends in detail how $\sigma_{i}$ depends on $\theta$, but for reasonable illustrative purposes, let us take $\sigma_{i}$ to be constant. Then, the integral in Eq. (C3) is simply $\sigma_{i} C_{i} \theta_{c}^{(i+2)} /(i+2)$, and, taking the terms in $n_{x}\left(\tau_{c}\right)$ to the left-hand side of Eq. (C3), we have

$$
\begin{aligned}
{\left[n_{x}\left(\tau_{c}\right)\right]^{(i+3)}=} & {\left[\sigma_{i} C_{i} /(i+2)\right] \cdot\left[\sigma_{x}\left(\tau_{c}\right)\right]^{-(i+2)} } \\
& \times\left(F / D_{1}\right)^{(i+1)} \exp \left(\beta E_{i}\right) .
\end{aligned}
$$

This result with scaling $(i+1) /(i+3)$ has been known for a long time (see, e.g., Ref. 10, p. 711, and the first of Ref. 12), and is not quite the same as the normal steady-state scaling $i /(i+2)$. This means that materials parameters can only be strictly separated out in the limit $i \rightarrow \infty$, when both laws become linear. These awkward, but minor, details will not concern us here. The primary question is what happens if the capture numbers are modified by attachment-limited kinetics?

First, we can see that if $\sigma_{x}\left(\tau_{c}\right)$ is attachment limited, as in Eq. (B3), then Eq. (C4) scales as $\left[2 \pi\left(r_{x}+1\right) \exp \left(-\beta E_{B}\right)\right]^{-(i+2)}$. This is a strong Arrhenius scaling, $n_{x}\left(\tau_{c}\right) \sim \exp \left[+\beta(i+2) E_{B} /(i+3)\right]$, dependent on $0.75 \beta E_{B}$ for $i=1$. The end of the transient regime can be $>1$ ML even for modest values of $\beta E_{B}$. Second, we can see that if both $\sigma_{i}$ and $\sigma_{x}$ are hindered by the same barrier, the situation considered by Kandel, ${ }^{19}$ the scaling goes as $\exp [+\beta(i$ $\left.+1) E_{B} /(i+3)\right]$, so that the important parameter is $0.5 \beta E_{B}$ for $i=1$, and the effect is still relatively strong in the same sense. Only in the case that $\sigma_{i}$ is hindered, but $\sigma_{x}$ is unhindered, does the effect go in the other direction, more weakly as $\exp \left[-\beta E_{B} /(i+3)\right]$. Thus, the effects of transient nucleation tend to undermine the quantitative comparisons with the experiment given in Ref. 19, based on steady-state expressions only. Illustrative examples for $i=1$ are given in Fig. 3. The effects are even stronger for larger- $i$ values, as noted previously in the case of no energy barriers. ${ }^{26}$

\section{APPENDIX D: BESSEL-TYPE EQUATIONS WITH NONCONSTANT COEFFICIENTS}

We are interested in solutions of a radial symmetric 2D diffusion equation for the adatom concentration $n_{1}(r)$ in the presence of a sink of radius $r_{k}$ at the origin with potential energy $V(r)$ around it. The steady-state diffusion solution with $V(r)=0$ is known (see Appendix A). The governing PDE is Eq. (9), and with the transformation $n_{1}$ $=n \cdot \exp [-\beta V(r)]$, Eq. (10) becomes the equation we need to solve for $n(r)$, with suitable boundary conditions, repeated here as Eq. (D1):

$$
\begin{aligned}
\nabla^{2} n(r)-n(r) /\left(D_{1} \tau\right)= & {[\partial n(r) / \partial t] / D_{1}+\nabla n(r) \cdot \beta \nabla V(r) } \\
& -\left(G / D_{1}\right) \cdot \exp [\beta V(r)] .
\end{aligned}
$$

Although the problem is, in principle, time dependent, we start from the time-independent formulation. Then, this is a standard Bessel function equation, except for two extra terms on the right-hand side. The steady-state solution for slowly varying $\left(D_{1} \tau\right)$, with $\tau=\tau_{0} \exp \{-\beta[V(r)-V]\}$ as explained below, is given in the text, leading to Eqs. (13) for $n_{1}$ and (14) for the capture number $\sigma_{k}$.

The purpose of this Appendix is

(i) to explore the next level of approximation for general 
$\left(D_{1} \tau\right)$ and $\exp [\beta V(r)]$, and to see if the slowly varying solution is likely to be valid for cases of practical interest;

(ii) to spell out algorithms for the diffusion-limited, attachment-limited, and interpolated capture numbers $\sigma_{k}$ for specific $\left(D_{1} \tau\right)$ and $\exp [\beta V(r)]$, which are used for the computational examples of Secs. IV and V.

\section{The slowly varying case and beyond}

The first approximation to Eq. (D1) arises from neglecting the first two terms on the right-hand side and treating the third term as a constant, from which the solution for $n(r)$ is as in Appendix A, namely,

$$
n(r)=A-B . K_{0}(X),
$$

with $X^{2}=r^{2} /\left(D_{1} \tau\right)$, where $A$ and $B$ are to be determined. The large- $r$ limit is just $n=G \tau \exp (\beta V)$. Since the large argument limit of $K_{0}(X)$ is $(\pi / 2 X)^{1 / 2} e^{-X}$, the second term in Eq. (D2), which accounts for the diffusive flux, goes exponentially to zero. Thus the term $A$ must account for $(G \tau) \cdot \exp [\beta V(r)]$ as well as possible over the important range of $r$. The uniform depletion approximation implies that $\tau$ is constant, but that is clearly inconsistent with the equilibrium case, as described in the text. If $\tau=\tau_{0} \exp [-\beta V(r)]$, then $A=(G \tau) \cdot \exp [\beta V(r)]=G \tau_{0} \exp (\beta V)$ is indeed constant, though $\tau$ varies. In the absence of a diffusion gradient then $n$ is constant, and $n_{1}(r)=n \cdot \exp [-\beta V(r)]$. With a diffusion gradient, this value of $n_{1}(r)$ is reduced to zero at $r=r_{k}$, but the diffusive contribution is reduced from the normal situation by the factor $\exp \left[-\beta V\left(r_{k}\right)\right]$. Converting Eq. (D2) back to $n_{1}(r)$ we have

$$
n_{1}(r)=G \tau-G \tau_{0} \exp \left[-\beta V\left(r_{k}\right)\right] . K_{0}(X) / K_{0}\left(X_{k}\right),
$$

as given in the text as Eq. (13), and Eq. (14) for the capture number follows from Eq. (8). The profile $n_{1}(r)$ for these equations is illustrated for a specific set of parameter values in Fig. 4(a).

Other approximations are possible, but they do not alter our qualitative conclusions. For example, when the capture numbers are small, transient nucleation is more important, and hence the first time-dependent term on the right-hand side of Eq. (D1) is not zero. In the limit of no nucleation $n_{1}(r)$ is initially equal to $G / D_{1}$, for all $r>r_{k}$. Since in this limit everything is linear, this term can then be combined with the third term, and cancels it precisely. But this is misleading, as the capture number results from the derivative at $r=r_{k}$ and this is mathematically infinite in such an initial situation, if only at $t=0$. Physically, the initial $\sigma_{k}$ is of order $2 \pi r_{k} \exp \left\{-\beta\left[V\left(r_{k}\right)-V\right]\right\}$ if $V(r)$ is reasonably short ranged. Over time, shorter or longer, depending on the parameter values, correlations develop with the diffusion solution becoming dominant.

The second approximation concerns the fact that the second term on the right-hand side of Eq. (D1) is not in general zero. This term depends on a product of derivatives $\nabla n(r) . \beta \nabla V(r)$, so if either $\nabla n(r)$ or $\nabla V(r)$ is zero this term vanishes, but it is zero both at large $r$, and when $V(r)$ has its maximum value near $r=r_{k}$, so Eq. (13) has the correct form in both these limits. However, this term is best incorporated into the left-hand side of Eq. (D1), such that it modifies the term $r^{-1} \partial n / \partial r$ contained within the 2D Laplacian by adding the positive term $-\beta \nabla V(r) \partial n / \partial r$, which can be significant in a small region where $r \nabla V(r)$ is numerically greatest. In effect, this term locally changes the shape of the Bessel function diffusion profile in the direction of becoming a modified spherical Bessel function of the third kind - the relevant solution of the 3D Laplacian, where the first-order term is $2 r^{-1} \partial n / \partial r .^{29}$ This solution has a simple analytic form, $(\pi / 2 X) e^{-X}$, which is somewhat smaller than $K_{0}(X)$ for $X>2$, and vice versa for $X<2$. Thus the shape of the $n_{1}(r)$ curve deviates from that discussed here by a small wobble at intermediate values of $X$, in the direction of making the curves nearer to step functions. But for the larger values of $V(r)$, including those illustrated in Fig. 4(a), the $n_{1}(r)$ curves are dominated by the equilibrium, constant $n(r)$ profile, except close to $r \sim r_{k}$; the diffusion component is sharply reduced, as emphasized in Eq. (13) and in the text discussion.

Finally, there is the possibility that $V(r)$ has its maximum value at $r>r_{k}$. Then, as shown by calculation of specific forms of $V(r)$, the assumed diffusion solution can exhibit unphysical negative values of $n_{1}(r)$. This arises, as explained in the text, because the assumed diffusive contribution is too large, and needs to be reduced to remain positive in the neighborhood of $r \sim\left(r_{k}+r_{0}\right)$. As found in Appendix B also, this case corresponds to attachment-limited kinetics, where we have to consider the atomistic events taking place around the maximum of $V(r)$. This is one of the special cases that have been pursued by computation, as illustrated in Fig. 4(b), and explained below.

\section{Capture number algorithms}

The algorithms used for diffusion and annealing programs have been explained in outline in Sec. V, and only a few points are elaborated here. The core of the codes is a simple, explicit, integration routine for $n_{1}, n_{x}$, and the coverage $\left(Z=n_{x} w_{x}\right)$, which contains the capture numbers $\sigma_{i}$ and $\sigma_{x}$. These few lines of code contain all the processes that lead to changes in $n_{1}, n_{x}$, and $Z$; the integration dose or time scale (linear, logarithmic, or square root as illustrated here) is set by a single " $\Delta t$ " parameter. Convergence of the code is easily checked by varying the step length by factors of 2 in either direction; smaller step lengths lead to better accuracy, provided digitally induced noise is avoided, but to larger file sizes; larger step lengths may decrease accuracy somewhat, but reduce file sizes markedly. In the cases illustrated here, file sizes were less than $100 \mathrm{kB}$, and files half this size would not markedly decrease the accuracy (differences less than the linewidth on the plots). More sophisticated integration routines are available in MATLAB ${ }^{\circledR} 5.3$, but are not really necessary.

The capture numbers themselves are iterated to selfconsistency at each dose or time step in a subroutine. For the case of an energy barrier $V(r)$, the central equations are Eq. (14) for both $\sigma_{i}$ and $\sigma_{x}$, with the arguments $X_{k}$ given by 


$$
X_{k}^{2}=r_{k}^{2}\left(f_{i} \sigma_{i} n_{1}+\sigma_{x} n_{x}\right)
$$

where $f_{i}=2$ for $i=1$, and $f_{i}=1$ otherwise. When the maximum of $V(r)$ occurs at $r=r_{0}+r_{k}$, the term in $r_{k}^{2}$ in Eq. (D4) is replaced by $\left(r_{0}+r_{k}-1\right)^{2}$. In this case, seen in Fig. 4(b), the approximate radial distribution $n_{1}(r)$, given otherwise by Eq. (13), has $\exp \left[-\beta V\left(r_{k}\right)\right]$ replaced by $\exp \left[-\beta V\left(r_{0}+r_{k}\right.\right.$ $-1)]$. Here the radial distribution is only valid for $r>\left(r_{0}\right.$ $\left.+r_{k}-1\right)$, and the corresponding attachment barrier $\left(\sigma_{B}\right)$ at $\left(r_{0}+r_{k}-1\right)$ is approximated by a single jump into the attractive region at smaller $r$.

These capture number routines require an initial estimate of $\sigma_{i}$ and $\sigma_{x}$, but it has been shown that the results are very insensitive to this initial choice; in other words, the differential equation integration is stable. Thus by starting the integration at a dose below the dose range of interest for the output, only small changes are introduced. The smoothness of the curves produced, and closeness to known analytical forms, allow us to choose suitable initial conditions.

There is an inherent uncertainty in choosing continuum boundary conditions to describe atomistic events, such as the radius of attachment barriers, or the exact area for direct impingement on monomers. For example, the continuum diffusion capture model will produce somewhat different capture numbers if the zero of concentration is at, say, 0.5 or 1 atomic radii outside the cluster, but such a distinction is at the edge of what is meaningful. For the deposition and annealing curves shown in Secs. IV and V, we have used a concentration zero at $\left(r_{k}+0.5\right)$ or $\left(r_{k}+1\right)$ in the absence of barriers, and with a barrier at $\left(r_{k}+r_{0}\right)$. Rather than overadjust these values to get exact agreement with KMC simulations or other atomistic simulations, we prefer to take a range of reasonable predictions into account when deducing material parameters from comparison with the model. ${ }^{25}$

\section{Interpolation formulae for time-dependent capture numbers}

As explained in outline in the text, an interpolation scheme is needed, which will take the value of the capture number from an initial uncorrelated value to a final diffusive value over the correct range. The capture number in this regime is a function of $\left(D_{1} t\right)$ and $B_{V}$ via Eq. (15), as can be seen from the rate equations for initial annealing with $G$ $=0$ :

$$
\begin{gathered}
d n_{1} / d\left(D_{1} t\right)=-2 \sigma_{1} n_{1}^{2}-\sigma_{x} n_{1} n_{x}, \\
d n_{x} / d\left(D_{1} t\right)=\sigma_{1} n_{1}^{2} .
\end{gathered}
$$

The initial conditions $\left(n_{1}, \sigma_{1}\right)$ and $\left(n_{x}, \sigma_{x}\right)$ for annealing are those appropriate to the end of deposition. In the lowtemperature, low dose, deposition case of $\mathrm{Cu} / \mathrm{Cu}(111)$ considered, there is very little diffusion during deposition, so that initial capture numbers are given by Eq. (15).

The coupled Eqs. (D5) and (D6) require time-dependent capture numbers, and one can see that, when $n_{1}>n_{x}$, the first term on the right-hand side of Eq. (D5) is likely to be larger than the second. By the time this situation is reversed, the diffusion solution will be appropriate, and the transient "problem" has gone away; the transient contains a finite number of adatoms nucleating and joining clusters. However, it is relatively more important during annealing than during deposition. During annealing there is no replacement of the adatom concentration, so if $\sigma_{1}$ is small via $\left(B_{V} / 2 \pi\right) \ll 1$, then $n_{1}$ stays at its initial value, and no annealing takes place. During deposition, however, the small values of $\sigma_{1}$ and $\sigma_{x}$ mean that the value of $n_{1}$ continues to increase, giving rise to a compensation effect. This effect can be seen at work in the schematic radial distribution curves shown in Fig. 4.

During annealing, however, the capture number decreases as follows:

$$
\sigma_{k}=\sigma_{\text {init }} f_{t}+\sigma_{k d}\left(1-f_{t}\right),
$$

where $\sigma_{k d}$ is the diffusive contribution given by Eq. (14), $\sigma_{\text {init }}$ is the initial barrier form of the capture number, and $f_{t}$ is a transient factor, such that at $\left(D_{1} t\right)=0, f_{t}=1$, and as $\left(D_{1} t\right) \rightarrow \infty, f_{t} \rightarrow 0$. Short of a complete closed-form solution to time-dependent equations for the capture numbers, we have experimented with justifiable forms of $f_{t}$. Our best fits to date are given in Figs. 6(b) and 7, based on $\sigma_{\text {init }}=\sigma_{B} f_{t}$, but note that this $f_{t}$ doesn't have to be the same as the $f_{t}$ in Eq. (D7). The physical argument used is that the transient is due to capture from a diffusion zone around the adatom or cluster considered, whose radius $r_{d}$ increases with time as some function of $\left(D_{1} t\right)$ and $B_{V}$.

In that case, we can write by analogy with Eq. (A1)

$$
f_{t}=K_{0}\left(X_{d}\right) / K_{0}\left(X_{k 0}\right) \text {, }
$$

where the new argument $X_{d}=\left(r_{k}+r_{0}+r_{d}\right) /\left(D_{1} \tau\right)^{1 / 2}$ and $X_{k 0}=\left(r_{k}+r_{0}\right) /\left(D_{1} \tau\right)^{1 / 2}$. In the case of $V_{0}$ or $E_{B}=0$, we know that $r_{d}$ will scale with $\left(D_{1} t\right)^{0.5}$. This range must be restricted when $V_{0}$ or $E_{B}>0$. If $n_{1}$ is initially constant during annealing, the detailed time dependence of $n_{x}$ in Eq. (D6) is given by $\sigma_{B}$, and this scales with a factor $\left(B_{V} / 2 \pi\right)$ directly. But also the initial value $\sigma_{\text {init }}$ decreases rapidly as diffusion over several lattice distances replaces attachment by a single jump. This feature, and the presence of square-law terms in Eqs. (D5) and (D6), qualitatively justifies the inclusion of the second factor $f_{t}$ in Eq. (16); this equation is just the simplest form that works.

Figures 6(b) and 7 show the near-perfect fit to the interpolation formula with

$$
r_{d}=\left(0.5 D_{1} t\right)^{0.5} B_{V} / 2 \pi
$$

The constant 0.5 in Eq. (D9) is the result of trial and error rather than detailed reasoning, and a full justification of Eqs. (D8) and (D9) may or may not merit further work. For the present the above equations give a reasonable interpolation scheme for making extrapolations to other temperatures, and for comparing with experiments. 
*Email address: john.venables@asu.edu

${ }^{1}$ J. A. Venables, Surf. Sci. 299/300, 798 (1994); Introduction to Surface and Thin Film Processes (Cambridge University, Cambridge, England, 2000), Chaps. 4 and 5.

${ }^{2}$ H. Brune, Surf. Sci. Rep. 31, 121 (1998).

${ }^{3}$ G. Ehrlich, Surf. Sci. 246, 1 (1991); 299/300, 628 (1994); in Surface Diffusion: Atomistic and Collective Phenomena, edited by M. Tringides (Plenum, New York, 1997), p. 23.

${ }^{4}$ T. T. Tsong, Atom-Probe Field Ion Microscopy (Cambridge University, Cambridge, England, 1990); G. L. Kellogg, Surf. Sci. Rep. 21, 1 (1994), and references therein.

${ }^{5}$ See, e.g., J. W. Martin and R. D. Doherty, Stability of Microstructure in Metallic Systems (Cambridge University, Cambridge, England, 1976), Secs. 2.4, 4.5, and 4.6; A. P. Sutton and R. W. Balluffi, Interfaces in Crystalline Materials (Oxford University, Oxford, England, 1995), Sec. 5.9, Chap. 10.

${ }^{6}$ J. Repp, F. Moresco, G. Meyer, K.-H. Rieder, P. Hyldgaard, and M. Persson, Phys. Rev. Lett. 85, 2981 (2000); for background information, a description of several systems where attachment barriers may be involved is given by J. V. Barth, H. Brune, B. Fischer, J. Weckesser, and K. Kern, ibid. 84, 1732 (2000). The case of $\mathrm{Al} / \mathrm{Al}(111)$ has not yet been settled; see T. Michely, W. Langenkamp, H. Hansen, and C. Busse, ibid. 86, 2695 (2000).

${ }^{7}$ N. Knorr, H. Brune, M. Epple, A. Hirstein, M. A. Schnieder, and K. Kern, Phys. Rev. B 65, 115420 (2002).

${ }^{8}$ A. Bogicevic, S. Ovesson, P. Hyldgaard, B. I. Lundqvist, H. Brune, and D. R. Jennison, Phys. Rev. Lett. 85, 1910 (2000).

${ }^{9}$ K. A. Fichthorn and M. Scheffler, Phys. Rev. Lett. 84, 5371 (2000)

${ }^{10}$ J. A. Venables, Philos. Mag. 27, 697 (1973), and references therein.

${ }^{11}$ J. A. Venables and D. J. Ball, Proc. R. Soc. London, Ser. A 322, 331 (1971); H. Schmeisser and M. Harsdorff, Philos. Mag. 27, 739 (1973).

${ }^{12}$ M. J. Stowell, Philos. Mag. 26, 349 (1972); 26, 361 (1972). Most calculations use one radial variable to define the "lattice" as a Wigner-Seitz "circle." An extensive numerical discussion is given by B. Lewis and G. J. Rees, ibid. 20, 1253 (1974) and by B. Lewis and J. C. Anderson, Nucleation and Growth of Thin Films (Academic, London, 1978), pp. 238-246.

${ }^{13}$ G. S. Bales and D. C. Chrzan, Phys. Rev. B 50, 6057 (1994).

${ }^{14}$ See Ref. 1, or J. A. Venables, Phys. Rev. B 36, 4153 (1987) for explicit expressions.

${ }^{15}$ J. G. Amar and F. Family, Phys. Rev. Lett. 74, 2066 (1995); M. C. Bartelt, C. R. Stoldt, C. J. Jenks, P. A. Thiel, and J. W. Evans, Phys. Rev. B 59, 3125 (1999); P. A. Mulheran and D. A. Robbie, Europhys. Lett. 49, 617 (2000); D. A. Robbie and P. A. Mulheran, Philos. Mag. B 80, 1299 (2000); J. G. Amar, M. N. Popescu, and F. Family, Phys. Rev. Lett. 86, 3092 (2001), and references therein.

${ }^{16}$ H. Brune, G. S. Bales, J. Jacobsen, C. Boragno, and K. Kern, Phys. Rev. B 60, 5991 (1999).

${ }^{17}$ Many papers in the 1990s used the approximation that capture numbers are constants; this is often not too inappropriate if only order-of-magnitude arguments are needed, or if only $n_{x}$ values for a single coverage are of interest. Other papers hinted at "logarithmic corrections," without specifying details. For ex- ample, at low coverage the uniform depletion approximation for $\sigma_{x}$ approximates to $4 \pi /[-\ln (Z)+0.116]$. But this is just the low- $Z$ limit of the full Bessel function solution, given in Ref. 10 and repeated here in Appendix A.

${ }^{18}$ F. G. Gibou, C. Ratsch, M. F. Gyure, S. Chen, and R. E. Caflisch, Phys. Rev. B 63, 115401 (2001).

${ }^{19}$ D. Kandel, Phys. Rev. Lett. 78, 499 (1997).

${ }^{20}$ S. Ovesson, A. Bogicevic, G. Wahnstrom, and B. I. Lundqvist, Phys. Rev. B 64, 125423 (2001).

${ }^{21}$ S. Ovesson, Phys. Rev. Lett. 88, 116102 (2002).

${ }^{22}$ K. H. Lau and W. Kohn, Surf. Sci. 75, 69 (1978). There are other effects with similar $r$ dependence, but for close-packed metals the surface-state effect appears to be the largest (see Refs. 7-9 for a discussion).

${ }^{23}$ This formulation is given, with different notation and different amounts of detail, in many places. See, for example, P. G. Shewmon, Diffusion in Solids (McGraw-Hill, New York, 1963), Secs. 1.5 and 4.4; R. Gomer, in Surface Mobilities on Solid Materials, Vol. 86 of NATO Advanced Studies Institute, Series B: Physics, edited by V. T. Binh (Plenum, New York, 1982), pp. 7-12; R. Gomer, Rep. Prog. Phys. 53, 917 (1990); A. R. Allnatt and A. B. Lidiard, Atomic Transport in Solids (Cambridge University, Cambridge, England, 1993), Secs. 5.2, 6.5, and 8.2.2.

${ }^{24}$ The activity of single adatoms is increased with repulsive interactions, $V(r)$, relative to Henry law adsorption, corresponding to a positive second virial coefficient in the adsorption isotherm (see Ref. 1, Chap. 4). The condition $\left(D / D^{*}\right)=\beta\{\delta \mu / \delta[\ln (c)]\}$ follows if there are "no cross-correlations between the velocities of different ad-particles" (see Ref. 23, R. Gomer, Rep. Prog. Phys., p. 926). The section in R. Gomer, Rep. Prog. Phys. (Ref. 23), pp. 927-928 shows that the diffusion constant involved in the Nernst-Einstein relationship is $D$, not $D^{*}$, and the formula (2.41) given for $\mathbf{j}(\mathbf{r})$ is stated in terms of the combination factor $D\{\beta \delta \mu / \delta[\ln (c)]\}^{-1}$. But this combination is just $D^{*}$ under the assumptions made here, so that formula (2.41) is the same as our Eq. (7a). Gomer has written out the formula for a nonideal adsorbed gas explicitly in Ref. 23, Surface Mobilities on Solid Materials, formula (22). In our application, the effects on $D$ and $D^{*}$ are probably secondary to the decrease in the concentration $n_{1}(r)$ due to $V(r)$ itself.

${ }^{25}$ To simplify the presentation, of Appendix B particularly, the units of length $r$ are normalized to the jump distance $a$, where $a^{-2}$ is the ML density. To be consistent, diffusion coefficient expressions are without a factor $a^{2}$ compared to conventional expressions. We have also noted in Appendices B and D, and in relation to Fig. 2, that there are uncertainties or order $0.5 a$ in the position of the zero in our continuum diffusion model. This is a rediscovery of the point published by C. Ratsch, M. Kang, and R. E. Caflisch, Phys. Rev. B 64, 020601 (2001) in the context of their level set models.

${ }^{26}$ Additional comments on effect of the critical nucleus size on transient nucleation are given by J. A. Venables, G. D. T. Spiller, and M. Hanbücken, Rep. Prog. Phys. 47, 399 (1984), pp. 410413. Scaling in the transition from $i=1$ to $i=0$ at low $\left(D_{1} / F\right)$ values, which is also a transient nucleation phenomenon, is thoroughly discussed in Ref. 16. 
${ }^{27}$ J. Jacobsen, K. W. Jacobsen, P. Stoltze, and J. K. Nørskov, Phys. Rev. Lett. 74, 2295 (1995).

${ }^{28}$ W. H. Press, S. A. Teukolsky, W. T. Vetterling, and B. P. Flannery, Numerical Recipes in C, 2nd ed. (Cambridge University, Cam- bridge, England, 1996), p. 282.

${ }^{29}$ Handbook of Mathematical Functions, edited by M. Abramowitz and I. A. Stegun [U.S. Department of Commerce (NBS, Appl. Math Series Vol. 55, 1970), Sec. 10.2. 